\title{
Propagation des ondes dans les lignes finies : discussion des notions d'onde évanescente et de fréquence de coupure
}

\author{
J. Kergomard (*) \\ Laboratoire d'acoustique, Université Paris VI, 4, place Jussieu, Tour 66, 75230 Paris Cedex 05, France
}

(Reçu le 5 mai 1981, révisé le 5 février 1982, accepté le 15 février 1982)

\begin{abstract}
Résumé. - Les problèmes de propagation des ondes dans les lignes se rencontrent, sous une forme ou sous une autre, dans de nombreux domaines de la physique. En particulier, les notions d'onde évanescente et de fréquence de coupure sont bien connues. Ce sont des notions idéales, définies sans ambiguité pour une ligne infinie sans pertes. On discute la généralisation de ces notions aux lignes finies. On montre ainsi que les ondes évanescentes demeurent décroissantes dans une ligne finie, sous certaine condition portant sur l'impédance terminale de la ligne; pour une ligne symétrique, cette condition porte simplement sur le signe de la partie imaginaire de cette impédance. Quant à la fréquence de coupure, dans les cas où elle peut être définie, elle dépend a priori de la longueur et de l'impédance terminale de la ligne. Cette discussion porte d'abord sur les lignes à caractéristiques constantes sur toute leur longueur, puis elle est étendue aux lignes à caractéristiques variables. Les exemples choisis justifient ou au contraire infirment des généralisations que notamment certains acousticiens ont admises comme évidentes.
\end{abstract}

\begin{abstract}
The problems of wave propagation in lines occur in one form or another in many fields of physics. The concepts of evanescent wave and cutoff frequency in particular are well known : they are ideal concepts, and are defined clearly only for an infinite line without losses. The paper attempts to extend those concepts to finite lines, and shows that evanescent waves remain decreasing for a finite line, under one condition about the terminal impedance of the line; for a symmetrical line, this condition refers to the sign of the imaginary part of that impedance. As regards cutoff frequency, when it can be defined, it depends on length and terminal impedance of the line. The study first considers the case of lines with constant parameters, then the case of lines with variable parameters. Choiced examples confirm or invalidate some generalizations which are sometimes considered to be obvious, in particular by acousticians.
\end{abstract}

1. Introduction. - 1.1 Propagation DES ONDES DANS LES LIGNES FINIES : POSITION DU PROBLÈME. - Il est bien connu en électricité que la notion de filtre est une notion idéale : il est en effet impossible pratiquement de réaliser de manière absolument rigoureuse un circuit qui atténue certaines fréquences et laisse passer les autres sans affaiblissement ni renforcement. Skilling [1] par exemple affirme que les calculs de filtres qu'il effectue comportent deux idéalisations : l'hypothèse que le filtre débouche sur son impédance image, et le fait de négliger les pertes de puissance.

Plus généralement, ceci est vrai des lignes, dont les filtres ne sont qu'un cas particulier défini par l'utilisation qui en est faite : les notions de fréquence de coupure, de bande passante, de bande d'arrêt, d'onde propagative et d'onde évanescente n'ont été définies à l'origine que dans le cas idéal d'une ligne sans pertes

$\left(^{*}\right)$ Adresse actuelle : Faculté des Sciences, Laboratoire d'Acoustique, Route de Laval, 72017 Le Mans Cedex, France. débouchant sur son impédance itérative (dite aussi caractéristique).

Il est à noter que celle-ci est obligatoirement réalisée à toute fréquence si la ligne est infiniment longue.

Par ailleurs, deux problèmes importants amènent à la même conclusion : d'une part, dans un guide d'onde, les modes non plans sont dits propagatifs ou évanescents suivant les fréquences; d'autre part, lors de la réfraction d'une onde oblique à l'interface de deux milieux différents, l'onde réfractée peut être propagative ou évanescente (dans la direction perpendiculaire à l'interface), suivant l'angle d'incidence. L'analogie de ces problèmes avec les lignes étant parfaite, la distinction entre onde propagative et onde évanescente n'est, là encore, nettement caractérisée que si le guide ou le deuxième milieu (pour l'onde réfractée) sont infinis.

Le problème de la généralisation des notions citées plus haut à une ligne quelconque (finie et débouchant sur une impédance quelconque) a déjà été abordé, quelquefois de manière explicite, mais le plus souvent, à propos d'applications pratiques, de manière impli- 
cite. La motivation de départ de cet article a été de relever certaines erreurs dans ces généralisations, rencontrées principalement en acoustique. Au-delà, nous visons à clarifier certaines propriétés, plus ou moins bien connues, des lignes finies : la transmission d'énergie dans ce que nous appellerons la bande d'arrêt idéale, la forme de l'amplitude des ondes dans cette bande d'arrêt idéale et les conditions de décroissance de l'amplitude avec la variable d'espace, la définition de la fréquence de coupure, ... Au préalable, nous devrons avoir défini précisément les différentes notions ; par ailleurs, il nous a semblé utile d'illustrer les propriétés mises en évidence par des exemples tirés de l'acoustique.

1.2 Les ÉTUDES ANTÉRIEURES SUR LE SUJET. - Les phénomènes de propagation des ondes sont d'une grande diversité : les auteurs qui les ont étudiés ont, pour la plupart, pensé aux nombreuses applications, et les études générales, comme l'ouvrage de L. Brillouin et M. Parodi [2] « Propagation des ondes dans les milieux périodiques ", sont assez rares.

Pourtant, dans la totalité de leur livre, ces auteurs sous-entendent que les lignes envisagées sont infinies ou suffisamment longues (à la page 73 de l'édition française, ils semblent en venir aux lignes finies, mais écrivent aussitôt : " $\mathrm{Si}$ nous voulons appliquer les résultats que nous avons obtenus à un réseau fini, il nous faut introduire aux extrémités libres des forces qui dépendent des conditions aux limites ").

Pour trouver des études concernant les lignes finies, nous avons donc dû rechercher des textes se rapportant aux diverses applications, ou aux problèmes analogues.

Ainsi, en optique, à propos du problème analogue de la réfraction d'une onde oblique à l'interface de deux milieux, le phénomène de réflexion totale sur le deuxième milieu peut disparaître si on rapproche un troisième milieu du premier. Ceci est connu depuis longtemps; citons par exemple Bouasse [3] : "On doit admettre que cette onde évanescente ne se propage pas au sens propre du mot; il faut la considérer comme un mouvement stationnaire, auquel nous pouvons conserver le nom d'onde et qui n'existe que jusqu'à une certaine profondeur (dans le deuxième milieu NDR). Son existence est indispensable pour qu'il y ait réflexion totale; si nous la supprimons par l'approche d'un autre corps, la réflexion totale n'a plus lieu. Ce n'est pas l'énergie (nulle d'après la théorie) de l'onde évanescente que nous recueillons, c'est le phénomène entier que nous modifions. " Quant à Bruhat [4] il évoque la réalisation d'une expérience qui « montre que la réflexion ne peut être totale que si la couche n'est pas trop mince ".

Plus généralement, la réflexion d'une onde sur un milieu stratifié (en optique et en acoustique) a été souvent étudiée. Ainsi Brekhovskikh, dans son livre "Waves in layered media " [5], étudie cette question et mentionne l'existence de fuite d'ondes à travers une couche finie : « Pour une couche infiniment épaisse, la réflexion totale interne se produit pour des angles d'incidence suffisamment grands. Cependant, avec une couche d'épaisseur finie, la réflexion ne peut pas se produire, et l'onde va pénétrer partiellement à l'intérieur de la couche. Cet effet est complètement analogue à la fuite d'une particule à travers une barrière de potentiel en mécanique quantique. "

A propos des filtres, les notions de bande d'arrêt, de bande passante et de fréquence de coupure ont été étendues aux cas pratiques : dans ce but, on avait d'abord cherché, pour une structure donnée, à réaliser au mieux sur la plus large bande de fréquence possible l'impédance itérative (cf. Campbell [6] et Johnson [7]); plus récemment, on a cherché, pour une courbe de perte d'insertion donnée, la structure de filtre qui convient le mieux (cf. Cauer [8], Collin [9], Temes et Mitra [10] par exemple). Il est à noter, en ce qui concerne les filtres mécaniques (chaînes d'oscillateurs couplés) et acoustiques, que Lindsay [11] a étendu la définition des ondes propagatives et évanescentes au cas d'un filtre débouchant sur l'impédance itérative des ondes planes.

Les ouvrages classiques traitant des guides d'ondes n'envisagent en général les notions de modes propagatifs et évanescents que pour des guides infiniment longs (les guides de longueur finie n'étant étudiés que pour les fréquences supérieures à la fréquence de coupure). Ces ouvrages portent aussi bien sur les ondes électromagnétiques que sur les ondes acoustiques (cf. par exemple [12-21]). Citons aussi dans ce cas le dictionnaire de l'I.E.E.E. [22]; celui-ci adopte une définition pour mode évanescent qui n'est valable que pour un guide infini : « Une configuration de champ... dont l'amplitude diminue le long du guide d'onde, mais la phase est inchangée "; par contre, il adopte une définition générale pour la fréquence de coupure : « La fréquence au-dessous de laquelle la constante de propagation est réelle $॥)$. A ce sujet, il est intéressant de citer De Broglie [23] à propos des guides d'ondes électromagnétiques rectangulaires : " $\mathrm{La}$ fréquence de coupure au-dessous de laquelle il n'y a pratiquement (souligné par nous) plus de propagation "; il sousentend vraisemblablement qu'il existe un problème pratique.

Pourtant, les effets analogues à "l'effet tunnel " de la mécanique quantique sont connus depuis assez longtemps et même utilisés par les spécialistes des guides d'ondes électromagnétiques : ainsi en est-il des filtres à résonateurs diélectriques [24], des filtres en guides à modes évanescents $[25,26]$, du couplage de deux guides d'ondes optiques [27], ...

Pour achever ce bref résumé (évidemment non exhaustif) des études antérieures sur notre sujet, nous évoquerons spécialement le problème des lignes à raccordements progressifs, notamment acoustiques : des calculs ont été faits concernant la propagation dans les pavillons de longueur finie (cf. [28-31]), mais le problème des ondes évanescentes dans les pavillons finis n'est jamais posé complètement. De plus, il l'est 
souvent de manière erronée : dans un pavillon exponentiel, la solution pour les ondes est de la forme

$$
p(x, t)=\left(a \mathrm{e}^{-k x}+b \mathrm{e}^{+k x}\right) \mathrm{e}^{j \omega t}
$$

dans cette expression, le rapport $b / a$ est complexe (que $k$ soit réel ou imaginaire pur), car déterminé par l'impédance à l'extrémité. De ce fait, quand $k$ est réel, le flux d'énergie transmis par ce pavillon n'est nul que si $b$ est nul, ou au moins si $b / a$ est réel : or plusieurs auteurs ont considéré que $b / a$ était toujours réel. C'est d'ailleurs la même erreur que commet Crawford [32] dans la résolution de l'équation de KleinGordon, qu'il effectue en grandeurs réelles : il obtient comme solution générale

$$
p(x, t)=\left(A \mathrm{e}^{-k z}+B \mathrm{e}^{+k z}\right) \cos (\omega t+\varphi),
$$

alors que celle-ci est en fait :

$$
A \mathrm{e}^{-k z} \cos (\omega t+\varphi)+B \mathrm{e}^{+k z} \cos \left(\omega t+\varphi^{\prime}\right) .
$$

1.3 Plan DE L'ÉtUde. - Le paragraphe 2 précise simplement les notations utilisées, en général bien connues, pour décrire les lignes, discrètes ou continues.

Dans le paragraphe 3, nous discutons les notions d'ondes propagatives et d'ondes évanescentes. Après avoir tenté de donner une définition précise à ces notions dans le cas idéal à l'aide de deux critères, nous cherchons si ceux-ci peuvent être étendus aux lignes finies sans pertes, et à quelles conditions.

Le paragraphe 4 discute l'existence de fréquences de coupure, et montre qu'il est très délicat d'obtenir des résultats généraux.

Le paragraphe 5 illustre les deux précédents, à l'aide de trois exemples : deux exemples de lignes symétriques (une onde réfractée dans un milieu homogène, et un mode non plan dans un guide), et un exemple de ligne asymétrique (le pavillon exponentiel).

Enfin le paragraphe 6 étend les résultats du paragraphe 3 aux lignes sans pertes à caractéristiques variables.

2. Description d'une ligne constituée de l'association de quadripôles en série : notations utilisées. - Nous voulons ici simplement préciser nos notations pour une ligne constituée de l'association de quadripôles identiques en série. Nous partirons de la ligne discrète, constituée de $n$ quadripôles. Puis nous étendrons les résultats à la ligne continue.

2.1 Relation matricielle. - Tout ce qui suit est écrit en nombres complexes. La grandeur réelle $P$ est donnée par $P=\operatorname{Re}\left(p \mathrm{e}^{j \omega t}\right)$, où $p$ est la grandeur complexe associée, $\omega$ la pulsation, $t$ le temps; le module de $p$ est l'amplitude de $P$, et l'argument de $p$, noté $\operatorname{Arg} p$, la phase de $P$ (on supposera toujours le régime permanent).

Soit un quadripôle défini par la relation suivante :

$$
\left\{\begin{array}{l}
p_{n}=B p_{n-1}+A u_{n-1} \\
u_{n}=D p_{n-1}+C u_{n-1}
\end{array} .\right.
$$

Les grandeurs $p$ et $u$ caractérisent complètement le champ dans la ligne envisagée : elles peuvent être par exemple la pression et une composante de la vitesse acoustiques, ou bien la tension et l'intensité électriques, ou toutes autres grandeurs (ainsi pour les pavillons acoustiques, on peut être amené à choisir des grandeurs abstraites, comme le produit du diamètre par la pression ou la vitesse acoustiques).

Les grandeurs $p$ et $u$ jouant des rôles équivalents, il peut être commode d'adopter la notation pour marquer une interversion de leurs rôles : ainsi $\bar{p}=u$ et $\bar{u}=p$; par contre, le conjugué d'un nombre $z$ sera toujours noté $z^{*}$.

L'indice $(n-1)$ est ici l'indice de l'entrée du $n$-ième quadripôle, et $n$ l'indice de la sortie de celui-ci (Fig. 1).

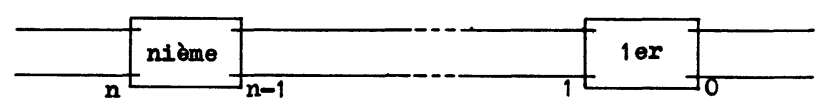

Fig. 1. - Numérotation des quadripôles.

[Numbers of the quadripoles.]

On suppose que la limite sur laquelle débouche la série de quadripôles est représentée par une condition d'impédance, telle que $Z_{0}=p_{0} / u_{0}$, où $Z_{0}$ est connue. En fonction de $p_{0}$ et $u_{0}$, on peut encore écrire :

$$
\left.\begin{array}{l}
p_{n}=B_{n} p_{0}+A_{n} u_{0} \\
u_{n}=D_{n} p_{0}+C_{n} u_{0}
\end{array}\right\}
$$

On suppose que $B C-A D=1$; si tel n'était pas le cas, $\left(B C-A D=\mathrm{e}^{2 W}\right)$ il suffirait de remplacer les variables $p_{n}$ et $u_{n}$ par $p_{n} / \mathrm{e}^{n W}$ et $u_{n} / \mathrm{e}^{n W}$, et les constantes $A, B, C$ et $D$ par $A / \mathrm{e}^{W}, B / \mathrm{e}^{W}, C / \mathrm{e}^{W}$ et $D / \mathrm{e}^{W}$.

Ceci posé, on trouve :

$$
\begin{cases}B_{n}=\cos n \phi+\Delta \frac{\sin n \phi}{\sin \phi} ; & A_{n}=A \frac{\sin n \phi}{\sin \phi} \\ D_{n}=D \frac{\sin n \phi}{\sin \phi} ; & C_{n}=\cos n \phi-\Delta \frac{\sin n \phi}{\sin \phi}\end{cases}
$$

où

$$
\cos \phi=\frac{B+C}{2} ; \Delta=\frac{B-C}{2},
$$

et donc

$$
A D=-\sin ^{2} \phi-\Delta^{2} .
$$

j $\phi$ est appelé constante de propagation du filtre (tous ces nombres sont complexes); on notera que $\phi$ est définie au signe près ; on choisira par convention toujours la valeur de $\phi$ dont la partie imaginaire est négative.

(Toutes les constantes $A, B, C, D, \zeta$ et $\phi$ sont en général fonctions de la fréquence, mais elles peuvent également dépendre d'autres paramètres.) 
2.2 RELATION HOMOGRAPHIQUE : IMPÉDANCE RAMENÉE ET IMPÉDANCE ITÉRATIVE. - On peut montrer que, si $Z=p / u$,

$$
Z_{n}=\frac{A_{n}+B_{n} Z_{0}}{C_{n}+D_{n} Z_{0}}
$$

peut se mettre sous la forme :

avec

$$
\zeta_{n}=\frac{-j \operatorname{tg} n \phi+\zeta_{0}}{1-j \operatorname{tg} n \phi 3_{0}}
$$

$$
3_{n}=\frac{Z_{n}}{\zeta+j \frac{\Delta}{\sin \phi}\left(Z_{n}-\zeta\right)}
$$

et

$$
\zeta=\frac{A}{-j \sin \phi-\Delta}=\frac{-j \sin \phi+\Delta}{D}
$$

$\zeta$ est appelée impédance itérative, c'est-à-dire impédance qui se reproduit égale à elle-même tout au long du filtre, et $Z_{n}$ est appelée impédance ramenée de $Z_{0}$ par $n$ éléments.

$\zeta$ est solution de l'équation $: \zeta=\frac{A+B \zeta}{C+D \zeta}$.

Cette équation a une autre solution : comme Brillouin, nous l'appellerons - $\zeta^{\prime}$, où $\zeta^{\prime}$ est l'impédance itérative « dans l'autre sens » :

$$
-\zeta^{\prime}=\frac{A}{j \sin \phi-\Delta}=\frac{j \sin \phi+\Delta}{D} .
$$

Nous y reviendrons au $\S 2.7$. Pour l'instant, nous en tirerons la forme canonique donnée par Brillouin à la relation matricielle :

$$
\left(\begin{array}{cc}
B_{n} & A_{n} \\
D_{n} & C_{n}
\end{array}\right)=\frac{1}{\zeta+\zeta^{\prime}}\left(\begin{array}{ll}
\zeta \mathrm{e}^{-j n \phi}+\zeta^{\prime} \mathrm{e}^{j n \phi} & -2 \zeta \zeta^{\prime} j \sin n \phi \\
-2 j \sin n \phi & \zeta^{\prime} \mathrm{e}^{-j n \phi}+\zeta \mathrm{e}^{j n \phi}
\end{array}\right) .
$$

Il en résulte :

$$
\zeta \zeta^{\prime}=\frac{A}{D}=\frac{A_{n}}{D_{n}}
$$

et

$$
3_{n}=\frac{Z_{n}\left(\zeta+\zeta^{\prime}\right)}{Z_{n}\left(\zeta-\zeta^{\prime}\right)+2 \zeta \zeta^{\prime}}
$$

$p$ et $u$ jouant des rôles identiques, on obtiendrait des relations similaires avec l'admittance $Y$, en interchangeant $A$ et $D$, et $B$ et $C$.

2.3 RELATION LINÉAIRE. - La relation matricielle peut également se mettre sous la forme :

$$
\left.\begin{array}{l}
p_{n}=a \mathrm{e}^{-j n \phi}+b \mathrm{e}^{j n \phi} \\
u_{n}=a / \zeta \mathrm{e}^{-j n \phi}-b / \zeta^{\prime} \mathrm{e}^{j n \phi}
\end{array}\right\}
$$

c'est-à-dire :

$$
\left\{\begin{array}{l}
p_{n}=a \mathrm{e}^{-j n \phi}+b \mathrm{e}^{j n \phi} \\
u_{n}=-\frac{\Delta}{A}\left(a \mathrm{e}^{-j n \phi}+b \mathrm{e}^{j n \phi}\right)-\frac{j \sin \phi}{A}\left(a \mathrm{e}^{-j n \phi}-b \mathrm{e}^{j n \phi}\right)
\end{array}\right.
$$

où $a$ et $b$ sont des constantes complexes déterminées par la connaissance de $p_{0}$ et $u_{0}$. Il est à noter que $b / a$, que Brillouin appelle coefficient de réflexion, est entièrement déterminé par $Z_{0}$; il vérifie :

$$
\frac{b}{a}=\mathrm{e}^{-2 j n \phi} \frac{Z / \zeta-1}{Z / \zeta^{\prime}+1}
$$

et donc en particulier :

$$
\frac{b}{a}=\frac{Z_{0} / \zeta-1}{Z_{0} / \zeta^{\prime}+1}
$$

$b$ est nul si $Z=\zeta$, et $a$ est nul si $Z=-\zeta^{\prime}$.

On ne retire rien à la généralité de la relation (3), en remarquant que toute ligne telle que $B C-A D=1$ peut être décrite par la relation (4) :

$$
\left.\begin{array}{l}
p=a \mathrm{e}^{-j n \phi}+b \mathrm{e}^{j n \phi} \\
u=\gamma\left(a \mathrm{e}^{-j n \phi}+b \mathrm{e}^{j n \phi}\right)+\delta\left(a \mathrm{e}^{-j n \phi}-b \mathrm{e}^{j n \phi}\right)
\end{array}\right\}
$$


on a

$$
\left\{\begin{array} { l } 
{ \gamma + \delta = 1 / \zeta } \\
{ \gamma - \delta = - 1 / \zeta ^ { \prime } }
\end{array} \text { ou } \left\{\begin{array}{l}
\gamma=\frac{1}{2}\left(\frac{1}{\zeta}-\frac{1}{\zeta^{\prime}}\right)=-\frac{\Delta}{A} \\
\delta=\frac{1}{2}\left(\frac{1}{\zeta}+\frac{1}{\zeta^{\prime}}\right)=-j \frac{\sin \phi}{A}
\end{array}\right.\right.
$$

La relation entre $Z_{n}$ et $Z_{n}$ (cf. $\S 2.2$ ) se met alors sous la forme :

$$
\frac{1}{Z_{n}}=\frac{\delta}{3_{n}}+\gamma
$$

En interchangeant les rôles de $p$ et $u$, on aurait de même :

où

$$
\left.\begin{array}{l}
u=\bar{a} \mathrm{e}^{-j n \phi}+\bar{b} \mathrm{e}^{j n \phi} \\
p=\bar{\gamma}\left(\bar{a} \mathrm{e}^{-j n \phi}+\bar{b} \mathrm{e}^{j n \phi}\right)+\bar{\delta}\left(\bar{a} \mathrm{e}^{-j n \phi}-\bar{b} \mathrm{e}^{j n \phi}\right)
\end{array}\right\}
$$

$$
\left\{\begin{array}{l}
\bar{\gamma}=\frac{1}{2}\left(\zeta-\zeta^{\prime}\right)=\frac{\Delta}{D} \\
\bar{\delta}=\frac{1}{2}\left(\zeta+\zeta^{\prime}\right)=-\frac{j \sin \phi}{D}
\end{array}\right.
$$

2.4 QUADRIPÔLE SYMÉTRIQUE (OU RETOURNABLE). Si $B=C$, c'est-à-dire si $\Delta$ (et $\gamma$ ) sont nuls, le quadripôle est dit symétrique (ou retournable). Les deux extrémités sont liées par la même relation, au signe de $A$ et $D$ près. Les deux impédances itératives sont alors égales.

\subsection{LES LIGNES CONTINUES À CARACTÉRISTIQUES} CONSTANTES. - La relation (3) relie les extrémités des différents quadripôles entre èlles; elle est donc valable pour des valeurs entières de $n$. Mais on peut l'étendre à tout nombre réel $X$ : on obtient ainsi une ligne continue, en général fictive, puisqu'il n'est pas possible, sauf cas très particuliers, de construire une ligne continue qui aurait les mêmes caractéristiques que la ligne discrète en certains points équidistants, correspondant aux extrémités des quadripôles. Ceci revient à assimiler le transfert à travers un quadripôle à une propagation.

- Inversement, on admettra avec Brillouin et Parodi que toute ligne continue peut être décrite par la relation suivante :

$$
\begin{aligned}
& p(x)=a \mathrm{e}^{-j \varphi x}+b \mathrm{e}^{j \varphi x} \\
& u(x)=a / \zeta \mathrm{e}^{-j \varphi x}-b / \zeta^{\prime} \mathrm{e}^{j \varphi x}
\end{aligned}
$$

Dans cette relation, $x$ est la coordonnée d'espace, $j \varphi$ la constante de propagation par unité de longueur, $\zeta$ et $\zeta^{\prime}$ les deux impédances itératives, $a$ et $b$ deux constantes quelconques. On peut également définir une relation analogue à la relation (4) et à la relation (4bis), en définissant $\gamma, \delta, \bar{\gamma}, \bar{\delta}$ à partir de $\zeta$ et $\zeta^{\prime}$, et de même une relation analogue à la relation matricielle canonique (1bis). Quant à la relation homographique (2), elle devient :

$$
3(x)=\frac{-j \operatorname{tg} \varphi x+3(0)}{1-\operatorname{tg} \varphi \times 3(0)}
$$

où

$$
\begin{aligned}
3(x) & =\frac{Z(x)\left(\zeta+\zeta^{\prime}\right)}{Z(x)\left(\zeta-\zeta^{\prime}\right)+2 \zeta \zeta^{\prime}} \\
Z(x) & =p(x) \mid u(x) .
\end{aligned}
$$

- A partir de cette ligne continue, on peut définir une infinité de lignes discrètes en choisissant une longueur $l$ pour le quadripôle de base; les relations matricielle (1) et homographique (2) sont alors obtenues de la façon suivante :

$$
\begin{aligned}
& \phi=\varphi l \quad A=-j \frac{\sin \phi}{\delta} \quad D=-j \frac{\sin \phi}{\bar{\delta}} \Delta=-\gamma A \\
& B=\cos \phi+\Delta \quad C=\cos \phi-\Delta .
\end{aligned}
$$

- La suite de l'exposé est écrite pour une ligne continue, mais il nous arrivera d'utiliser parfois aussi les notations valables pour une ligne discrète, les relations ci-dessus permettant le passage de l'une à l'autre; l étant une longueur quelconque, les grandeurs sans dimension $\phi=\varphi l$ et $X=x / l$ correspondant à la ligne discrète, et les grandeurs $\varphi$ (inverse d'une longueur) et $x$ (longueur) correspondant à la ligne continue.

Pour la ligne continue, on peut ajouter une notation différentielle aux autres notations. Pour la trouver, on peut : 
- soit partir de la relation matricielle de la ligne discrète, et passer à la limite : (1) peut s'écrire :

$$
\left\{\begin{array}{l}
p_{n}=\underline{A} u_{n}+\underline{B}\left(u_{n}-u_{0}\right) \\
u_{n}=\underline{C} p_{n}+\underline{D}\left(p_{n}-p_{0}\right)
\end{array}\right.
$$

- soit plus simplement partir de la notation linéaire (4) et (4bis) : on obtient :

$$
\left.\begin{array}{l}
u=\gamma p+j \frac{\delta}{\varphi} p^{\prime} \\
p=\bar{\gamma} u+j \frac{\bar{\delta}}{\varphi} u^{\prime}
\end{array}\right\}
$$

(dans tout ce qui suit, ' et " représenteront toujours les première et deuxième dérivations par rapport à $x$ )

2.6 CAS ou $B C-A D=\mathrm{e}^{2 W} \neq 1$. - Dans ce cas, la relation (4) s'écrit :

$$
\left.\begin{array}{l}
p_{n}=\mathrm{e}^{n W}\left(a \mathrm{e}^{-j \phi n}+b \mathrm{e}^{j \phi n}\right) \\
u_{n}=\gamma p_{n}+\delta \mathrm{e}^{n W}\left(a \mathrm{e}^{-j \phi n}-b \mathrm{e}^{j \phi n}\right)
\end{array}\right\}
$$

où $\cos \phi=\frac{1}{2}(B+C) \mathrm{e}^{-W} ; \gamma=-\frac{\Delta}{A}$;

$$
\delta=-j \frac{\sin \phi}{A} \mathrm{e}^{W}
$$

Le passage de la ligne discrète à la ligne continue s'effectue dans les conditions indiquées au $\S 2.5, W$ étant à remplacer par $w$, inverse d'une longueur. Le système différentiel (5) s'écrit alors :

$$
\left.\begin{array}{l}
u=\left(\gamma-j \frac{\delta}{\varphi} w\right) p+j \frac{\delta}{\varphi} p^{\prime} \\
p=\left(\bar{\gamma}-j \frac{\bar{\delta}}{\varphi} w\right) u+j \frac{\bar{\delta}}{\varphi} u^{\prime}
\end{array}\right\}
$$

2.7 EQUIVALENCE LIGNE INFINIE-LIGNE DÉBOUCHANT SUR SON IMPÉDANCE ITÉRATIVE. - 2.7.1 Si $\varphi$ n'est pas réel. - Si l'extrémité 0 est rejetée à l'infini, la relation (3) montre alors que :

Soit

$$
p \sim a \mathrm{e}^{-j \varphi x}, u \sim a \mathrm{e}^{-j \varphi x} / \zeta, \quad \text { et } Z \rightarrow \zeta
$$

Soit

$$
p \sim b \mathrm{e}^{j \varphi x}, \quad u \sim b \mathrm{e}^{j \varphi x} /-\zeta^{\prime}, \quad \text { et } \quad Z \rightarrow-\zeta^{\prime} .
$$

Par convention, nous supposerons que l'axe des $x$ est orienté en partant de la source $B$ (Fig. 2) :
0

Fig. 2. - Convention d'orientation.
Or nous avons choisi $\varphi$ tel que $\operatorname{Im}(\varphi)<0$; par conséquent les grandeurs ne pouvant devenir infinies quand $x$ croît, seule la première éventualité de ligne infinie nous intéresse : $\zeta$ sera l'impédance itérative que nous retiendrons, et $a$ ne sera jamais nul, puisque imposé par la source.

2.7.2 Si $\varphi$ est réel. - Il n'y a alors plus convergence vers 0 des exponentielles quand $x$ tend vers l'infini. Mais ce cas n'a pas de réalité physique stricte, puisqu'il impliquerait l'inexistence de pertes. Or si celles-ci peuvent être négligées localement, elles ne peuvent pas l'être sur une longue distance.

Par conséquent, pour une ligne infinie (c.-à-d. très longue), dans une portion très éloignée de l'extrémité, il est possible, tout en négligeant les pertes, d'admettre que l'impédance est toujours l'impédance itérative, que $\varphi$ soit réel ou non.

\section{Amplitude des ondes dans les lignes sans pertes. -} 3.1 CONDITION IMPOSÉE À UNE LIGNE SANS PERTES. Le flux moyen d'énergie transmis d'un quadripôle à l'autre est donné par la relation : $I=\frac{1}{2} \operatorname{Re}\left(p u^{*}\right)$.

La ligne est dite sans pertes (pertes sous forme calorifique : amortissement; pertes sous forme d'énergie de même nature que les vibrations considérées : rayonnement) si le flux ne varie pas d'un quadripôle à l'autre; pour une ligne continue, il doit donc être indépendant de $x$.

Ceci impose trois conditions aux coefficients du quadripôle : $A C^{*}$ imaginaire pur, $B D^{*}$ également, et $B C^{*}+A^{*} D=1$.

Ceci implique : $A B^{*}$ et $C D^{*}$ imaginaires purs, et $\left|\mathrm{e}^{2 W}\right|=|B C-A D|=1$.

Si on ajoute la condition $W=0$, le flux d'énergie est constant si et seulement si $B$ et $C$ sont réels, et $A$ et $D$ sont imaginaires purs. Le système $(6) \mathrm{du} \S 2.6$ nous montre que si on n'impose pas la condition $W=0$, seule la phase des grandeurs sera affectée, mais non leur amplitude. Il était au demeurant évident que la condition $\operatorname{Re}\left(p u^{*}\right)$ constante est inchangée si on multiplie $p$ et $u$ par la même constante complexe de module 1.

On obtient alors : $\cos \phi=\cos \varphi l$ réel, et

$$
\gamma=\frac{1}{2} \frac{C-B}{A} \text { et } \bar{\gamma}=\frac{1}{2} \frac{B-C}{D}
$$

imaginaires purs. Deux cas se présentent : soit $\cos \varphi l<1$, et

$$
\left\{\begin{array}{l}
\varphi \text { réel } \\
\zeta \text { complexe, avec } \operatorname{Re}(\zeta) \neq 0, \text { et } \zeta=\zeta^{\prime *} \\
(\zeta \text { réel si le filtre est symétrique) } \\
\delta=-\frac{j \sin \varphi l}{A} \text { et } \bar{\delta}=-\frac{j \sin \varphi l}{D} \text { réels }
\end{array}\right.
$$

soit $\cos \varphi l>1$, et

$$
\left\{\begin{array}{l}
\varphi \text { imaginaire pur } \\
\zeta \text { et } \zeta^{\prime} \text { imaginaires purs } \\
\delta \text { et } \delta \text { imaginaires purs . }
\end{array}\right.
$$

[Conventional orientation.] 
Remarque : comme nous l'avons dit (cf. § 2.7.2), il n'est pas possible de négliger les pertes sur une longue distance. Mais à notre avis, dans bien des cas, les pertes sont faibles, et les phénomènes décrits ne sont pas qualitativement modifiés. Certes, en toute rigueur, il conviendrait de traiter par exemple des ondes "quasi évanescentes", selon l'expression de Jouguet [33]. Ceci ne nous semble pas indispensable; c'est pourquoi nous limiterons l'ensemble de l'étude qui suit aux lignes sans pertes, même si nous n'oublions pas le caractère idéal de telles lignes.

Nous montrons en annexe I que le flux d'énergie peut se mettre sous la forme suivante,

$$
I=-\frac{1}{2} \frac{\delta}{\varphi}|p|^{2}[\operatorname{Arg} p]^{\prime}=-\frac{1}{2} \frac{\bar{\delta}}{\varphi}|u|^{2}[\operatorname{Arg} u]^{\prime} .
$$

Il est proportionnel au carré de l'amplitude et à la dérivée de la phase de chacune des grandeurs. Pour caractériser des ondes, on peut donc se contenter par exemple de l'amplitude et du flux d'énergie, la variation de phase n'apportant pas d'informations supplémentaires. Ainsi, il est équivalent de dire que le flux d'énergie est nul ou qu'il n'y a pas de rotation de phase (ceci correspond au cas des ondes stationnaires, pour lesquelles les grandeurs réelles peuvent se mettre sous la forme $f(x) g(t)$ : ceci est la définition la plus couramment admise pour ce terme, et nous l'adopterons).

3.2 Le FILTRE IDÉAL : LIGNE SANS PERTES DÉBOUCHANT SUR SON IMPÉDANCE ITÉRATIVE. On a (cf. § 2.6) :

$$
\left\{\begin{array}{l}
p=a \mathrm{e}^{-j \varphi x} \\
u=a / \zeta \mathrm{e}^{-j \varphi x}
\end{array}\right.
$$

Si $\varphi$ est réel :

$$
\left\{\begin{array}{l}
|p|^{2}=|a|^{2} \\
|u|^{2}=\frac{|a|^{2}}{|\zeta|^{2}} .
\end{array}\right.
$$

Si $\varphi$ est imaginaire pur (soit $\varphi=-j \alpha$, où $\alpha$ est positif) :

$$
\left\{\begin{array}{l}
|p|^{2}=|a|^{2} \mathrm{e}^{-2 \alpha x} \\
|u|^{2}=|a|^{2} /|\zeta|^{2} \mathrm{e}^{-2 \alpha x}
\end{array}\right.
$$

On a donc réalisé un filtre en $p$ et $u$ :

Dans la bande passante ( $\varphi^{2}$ positif), $\left|\frac{p}{p_{0}}\right|$ et $\left|\frac{u}{u_{0}}\right|$ sont constants et égaux à 1 .

Dans la bande d'arrêt ( $\varphi^{2}$ négatif), $\left|\frac{\mathrm{p}}{p_{0}}\right|$ et $\left|\frac{u}{u_{0}}\right|$ sont supérieurs à 1 et décroissent exponentiellement dans l'espace.

Ces deux bandes sont séparées par la valeur critique $(\varphi=0)$ de la constante de propagation, pour laquelle $\left|\frac{p}{p_{0}}\right|$ et $\left|\frac{u}{u_{0}}\right|$ sont constants et égaux à 1 .
On peut trouver deux critères de différenciation des deux bandes, (à $\varphi$ constant) :

1) Le critère d'amplitude : dans la bande passante, l'amplitude est constante le long de la ligne. Dans la bande d'arrêt, elle décroît exponentiellement. Les ondes sont alors dites évanescentes. Certains auteurs parlent dans ce cas d'atténuation réactive des ondes [1].

2) critère de flux d'énergie : celui-ci est non nul dans la bande passante, et nul dans la bande d'arrêt. Comme nous l'avons noté, ceci revient aussi à dire que la phase varie dans la bande passante, et est constante dans la bande d'arrêt.

Au sens strict, les ondes évanescentes sont donc un cas particulier d'ondes stationnaires qui décroissent exponentiellement avec la coordonnée d'espace.

Un troisième critère est souvent utilisé pour distinguer les deux bandes : c'est celui de la périodicité dans l'espace. Il découle des deux critères précédents, mais ne s'applique en toute rigueur qu'aux lignes infinies : dans la bande passante, les ondes sont périodiques dans l'espace, et dites alors propagatives : au contraire, elles ne le sont pas dans la bande d'arrêt. Pour pouvoir définir des ondes propagatives pour une ligne finie débouchant sur son impédance itérative, il faudrait le faire avec un critère d'amplitude, ou, mieux, avec un critère portant sur la variation de la phase. Nous définirons une onde propagative comme une onde dont la phase varie linéairement avec $x$, autrement dit telle que la dérivée de la phase est constante. Ceci implique, pour les lignes sans pertes, une amplitude constante.

Nous avons choisi le terme propagatif, distinct de progressif, car les diverses définitions de ce dernier terme sont en général beaucoup plus restreintes que la définition ci-dessus, et ne peuvent pas s'appliquer à certaines lignes très particulières, comme les filtres, les pavillons, ...

Les définitions précedentes correspondent au cas idéal d'une ligne débouchant sur son impédance itérative : il nous arrivera dans la suite de l'exposé d'employer l'expression bande passante idéale, bande d'arrêt idéale, ou valeur critique idéale, pour désigner la bande passante, la bande d'arrêt ou la valeur critique de $\varphi$ dans le cas d'une ligne idéale.

3.3 LigNe (FINIE) SANS PERTES NE DÉBOUCHANT PAS SUR SON IMPÉDANCE ITÉRATIVE. - 3.3.1 Difficulté de la généralisation. - Si $b$ n'est pas nul, on a :

$$
\begin{aligned}
& p=a \mathrm{e}^{-j \varphi x}+b e^{j \varphi x} \\
& u=a / \zeta \mathrm{e}^{-j \varphi x}-b / \zeta^{\prime} \mathrm{e}^{j \varphi x}
\end{aligned}
$$

avec $\varphi$ réel ou imaginaire pur, et $b / a$ en général complexe, car déterminé par $Z_{0}$ (cf. § 2.3).

Aucun des deux critères de distinction entre les deux cas $\varphi$ réel et $\varphi$ imaginaire pur ne peut être conservé :

Pour le critère d'amplitude : si $\varphi$ est réel, l'amplitude oscille entre des extremums (nœuds et ventres). Si $\varphi$ 
est imaginaire pur, l'amplitude peut décroître ou croître, comme nous le montrerons au $\S 3.3 .4$; pour le critère de flux d'énergie : si $\varphi$ est imaginaire pur, $I=j \delta \operatorname{Im}\left(a b^{*}\right)$ (cf. annexe I); il est donc non nul, sauf si $b / a$ est réel. De plus, si $\varphi$ est réel,

$$
I=\frac{1}{2} \operatorname{Re}(1 / \zeta)\left(|a|^{2}-|b|^{2}\right)
$$

peut s'annuler si $|b|=|a|$. Que $\varphi$ soit réel ou imaginaire pur, le flux d'énergie peut aussi bien être nul que non nul (autrement dit, la phase peut aussi bien varier qu'être constante).

On peut conclure que la généralisation du cas idéal au cas général est a priori abusive; ainsi que nous l'avons mentionné dans l'introduction, cette affirmation est connue à propos d'un certain nombre d'applications, mais pour d'autres, comme le pavillon acoustique exponentiel, on rencontre des erreurs : de fait, les rares auteurs qui ont abordé le problème du pavillon fini se sont en général trompés.

C'est ainsi que Rocard [30,31] généralise le deuxième critère, affirmant que tous les points vibrent en phase en dessous de la fréquence de coupure; Drawin [34] affirme qu'il y a alors "superposition d'ondes stationnaires " (au sens où nous les avons définies $\S 3.1)$ : ceci n'est certes pas faux, mais il aurait dû préciser que cette superposition d'ondes stationnaires n'est en général pas elle-même une onde stationnaire. Quant à Kinsler et Frey [35], ils ont observé expérimentalement que le flux d'énergie n'est pas nul : ils en déduisent que les prémisses de la théorie sont fausses, alors qu'en fait seule la généralisation dont nous avons parlé est fausse. Seul Olson remarque [29] : « Le pavillon exponentiel, bien sûr, transmet au-dessous de cette fréquence (de coupure), parce que la résistance acoustique n'est pas nulle; cependant, sauf dans le cas où la gorge est comparable à la bouche, la valeur de la résistance acoustique pour les fréquences inférieures à la fréquence de coupure, est assez petite. " Nous y reviendrons de manière plus approfondie; nous montrerons notamment que Olson a raison car le pavillon qu'il étudie est tel que l'impédance de rayonnement est très inférieure en module à l'impédance itérative des ondes planes, pour les fréquences inférieures à la fréquence de coupure du pavillon infini (cf. $\S 5.3$ ).

\subsubsection{Les variations dans l'espace de l'amplitude} des ondes. - Le critère de flux d'énergie ne permet pas de généralisation simple; par contre, nous allons montrer que le critère d'amplitude peut dans certains cas être modifié pour permettre une distinction entre deux types d'ondes. En effet, l'amplitude des ondes dans la bande d'arrêt idéale peut demeurer une fonction décroissante à concavité positive, sous certaines conditions imposées à l'impédance terminale $Z_{0}$.

Nous nous proposons donc d'étudier les fonctions $|p(x)|$ et $|u(x)|$. On notera que leurs variations ne sont pas forcément identiques : c'est une des raisons pour lesquelles il nous semble plus complet de ne pas nous limiter à une forme d'énergie (potentielle ou cinétique par exemple) mais de nous intéresser aussi à l'énergie totale. Celle-ci représente souvent les phénomènes de façon plus globale; ainsi une onde plane stationnaire présente de fortes variations d'amplitude de $p$ et $u$, mais l'énergie totale est constante dans l'espace.

Pour étudier à la fois les variations de ces trois énergies, nous étudierons la fonction d'amplitude $e(x)=L^{2}|p|^{2}+M^{2}|u|^{2}$, où $L$ et $M$ sont des constantes réelles connues dans chaque cas : en choisissant $L$ nul ou $M$ nul, on obtient respectivement la densité d'énergie cinétique $e_{\mathrm{c}}(x)$ ou potentielle $e_{\mathrm{p}}(x)$, et, si $L$ et $M$ sont non nuls, la densité d'énergie totale $e_{\mathrm{t}}(x)$.

\subsubsection{Etude de la fonction amplitude.-}

$$
e(x)=L^{2}|p|^{2}+M^{2}|u|^{2} .
$$

Dans ce qui suit, nous emploierons selon les besoins l'une ou l'autre des différentes notations définies au § 2. (Le détail des calculs se trouve en annexe I.) Rappelons que l'indice 0 représente l'extrémité à droite, où l'impédance $Z_{0}$ est connue; 0 est aussi l'origine, par conséquent $x$ est partout négatif dans la ligne.

3.3.3.1 Si $\varphi$ est réel (bande passante idéale). Avec les notations du $\S 2.3$, on peut écrire :

$$
\begin{aligned}
e= & \left(|a|^{2}+|b|^{2}\right)\left(L^{2}+M^{2} /|\zeta|^{2}\right)+2|a b| \times \\
& \times\left[L^{2} \cos (2 \varphi x-\theta)-M^{2} /|\zeta|^{2} \cos \left(2 \varphi x-\theta^{\prime}\right)\right]
\end{aligned}
$$

où

$$
\begin{aligned}
& \theta=\arg \left(a b^{*}\right) \text { et } \theta^{\prime}=\arg \left(a b^{*} / \zeta^{2}\right) \\
& e^{\prime}=-4 \varphi|a b| \times \\
& \times {\left[L^{2} \sin (2 \varphi x-\theta)-M^{2} /|\zeta|^{2} \sin \left(2 \varphi x-\theta^{\prime}\right)\right] } \\
& e^{\prime \prime}=-8 \varphi^{2}|a b| \times \\
& \times\left[L^{2} \cos (2 \varphi x-\theta)-M^{2} /|\zeta|^{2} \cos \left(2 \varphi x-\theta^{\prime}\right)\right]
\end{aligned}
$$

$e$ est une fonction périodique dans l'espace, de période $\pi / \varphi$. Si $b$ est nul (ligne débouchant sur son impédance itérative), $e$ est constante ; elle l'est également dans un cas particulier, si $\zeta$ est réel et $L=M /|\zeta|$ (c'est le cas de l'énergie totale d'une onde plane).

$e$ est une sinusoîde : comme la ligne est finie, ses extrémités peuvent être situées à un endroit quelconque d'une période, et par conséquent $e_{0}^{\prime}$ et $e_{0}^{\prime \prime}$ peuvent être aussi bien négatives que positives ou nulles. Quand $\varphi$ tend vers 0 , la période tend vers l'infini, et devient donc plus longue que la ligne : la courbe de $e$ est alors une simple portion de sinusoïde, de sens de variation et de concavité quelconques. Cela dépend de $\theta$ et $\theta^{\prime}$, c'est-à-dire de $\arg (b / a)$ et $\arg \left(b / a \zeta^{2}\right)$, soit $\arg \left(\frac{Z_{0} / \zeta-1}{Z_{0} / \zeta^{\prime}+1}\right)$ et $\arg \left(\frac{Z_{0} / \zeta-1}{Z_{0} / \zeta^{\prime}+1} \frac{1}{\zeta^{2}}\right)$. 


\subsubsection{2 $\mathrm{Si} \varphi$ est imaginaire pur (bande d'arrêt}

idéale. - Avec les notations du $\S 2.1$, on peut écrire, $\overline{\operatorname{si} j \varphi}=\alpha$, où $\alpha$ positif et $j \phi=\alpha l$ :

$$
\begin{aligned}
e=\sinh ^{2} \alpha x\left(|U|^{2}+|V|^{2}\right)+2 & \operatorname{Re}\left(U V^{*}\right) \times \\
& \times \sinh 2 \alpha x+e_{0}
\end{aligned}
$$

où

$$
U=\frac{(L A-M \Delta) u_{0}+(M D-L \Delta) p_{0}}{\sinh \alpha l}
$$

et

$$
V=L p_{0}+M u_{0} .
$$

Les dérivées de $e$ par rapport à $x$ sont :

$$
\begin{aligned}
e^{\prime}=\left[\operatorname { s i n h } 2 \alpha x \left(|U|^{2}+\mid\right.\right. & \left.\left.V\right|^{2}\right)+ \\
& \left.+2 \cosh 2 \alpha x \operatorname{Re}\left(U V^{*}\right)\right] \\
e^{\prime \prime}=2 \alpha^{2}\left[\operatorname { c o s h } 2 \alpha x \left(|U|^{2}\right.\right. & \left.+|V|^{2}\right)+ \\
& \left.+2 \sinh 2 \alpha x \operatorname{Re}\left(U V^{*}\right)\right] .
\end{aligned}
$$

On montre (cf. annexe 1) que $e^{\prime \prime}$ est strictement positive quel que soit $x$; la fonction amplitude présente donc au plus un minimum, et il y a trois cas de figure possibles (Fig. 3).
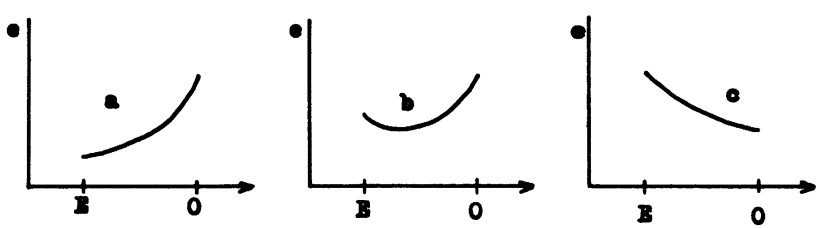

Fig. 3. - Variations possibles de $e(x)$ dans la bande d'arrêt idéale.

[Possible variations of $e(x)$ in ideal stop band.]

Comme on le voit, les ondes ne demeurent monotones décroissantes, comme dans le cas idéal, que dans le cas c. On peut donc considérer, en suivant le sens éthymologique du terme " évanescent ", que dans les autres cas les ondes ne sont pas évanescentes. Mais une définition élargie de ce terme peut également se concevoir, une onde évanescente devenant une onde telle que la constante de propagation soit réelle. Au-delà du choix des définitions, il est intéressant de savoir à quelle condition les ondes sont décroissantes : celle-ci est donnée par le signe de la dérivée de $e$ en 0 (c'est-à-dire à la sortie), qui permet de distinguer le cas c des autres cas. Or $e_{0}^{\prime}$ a le signe de la quantité suivante, écrite successivement avec les notations du $\S 2.1$ et du $\S 2.3$ (si $Y_{0}$ est l'admittance de sortie) :

$$
\begin{aligned}
\Delta\left(L^{2}-M^{2}\left|Y_{0}\right|^{2}\right)+j\left(L^{2} A-M^{2} D\right) \operatorname{Im}\left(Y_{0}\right) & \\
\alpha \frac{\gamma}{\delta}\left(L^{2}-M^{2}\left|Y_{0}\right|^{2}\right)+j \alpha \times & \\
\times & {\left[-\frac{L^{2}}{\delta}+M^{2} \delta\left(1-\frac{\gamma^{2}}{\delta^{2}}\right)\right] \operatorname{Im}\left(Y_{0}\right) }
\end{aligned}
$$

où $\alpha$ et $\Delta$ sont réels et $A, D, \gamma$ et $\delta$ sont imaginaires purs.
Un cas très remarquable est celui du quadripôle symétrique : si $\gamma=0, e_{0}^{\prime}$ a le signe de $j \delta \operatorname{Im}\left(Y_{0}\right)$. Celui-ci ne dépend donc pas de $L$ ni de $M$. Autrement dit, les énergies totale, potentielle et cinétique évoluent toujours dans le même sens. La condition de décroissance est en particulier remplie dans le cas où $Y_{0}$ est réelle : nous retrouvons ainsi, de manière beaucoup plus générale, le résultat obtenu par Lindsay pour une ligne symétrique débouchant sur l'impédance itérative des ondes planes.

Si on avait cherché les conditions sur le « coefficient de réflexion $"|b / a|$ (cf. $\S 2.3$ ), on aurait trouvé pour le cas $c$ :

$$
\left|\frac{b}{a}\right|^{2}<\frac{L^{2}+M^{2} /|\zeta|^{2}}{L^{2}+M^{2} /\left|\zeta^{\prime}\right|^{2}}
$$

On retrouve la simplicité du quadripôle symétrique $\left(\zeta=\zeta^{\prime}\right)$

$$
\left|\frac{b}{a}\right|^{2}<1 \text {. }
$$

Remarque : nous avons déjà montré que $b / a$ n'est pas réel en général. De plus, $|b / a|$ n'est pas forcément inférieur à 1 , contrairement à la bande passante idéale. Dans celle-ci en effet, le flux d'énergie s'écrit $I=\frac{1}{2} \operatorname{Re}(1 / \zeta)\left(|a|^{2}-|b|^{2}\right) ;$ d'après la position choisie pour la source (\$ 2.6), le flux d'énergie doit être positif, quel que soit $b$, et donc $|b|$ inférieur à $|a|$. Par contre, il n'en est rien dans la bande d'arrêt idéale.

A ce propos, il est souvent utile de s'assurer qu'une ligne est suffisamment longue pour que les ondes soient négligeables à la sortie. Intuitivement, on pourrait penser qu'il suffit que $-\alpha x$ soit assez grand pour que le terme $b \mathrm{e}^{\alpha x}$ soit négligeable ( $p$ s'écrit $\left.p=a \mathrm{e}^{-\alpha x}+b \mathrm{e}^{\alpha x}\right)$. En fait, on supposerait alors que $|b / a|$ est petit, ce qui n'est pas du tout évident. Il est donc insuffisant de connaître la longueur d'une ligne pour trouver à quelle condition les ondes sont négligeables à la sortie : il faut de plus connaître l'impédance de sortie. Ceci vaut en particulier pour les modes non plans d'un guide : pour pouvoir négliger un mode dit évanescent (au sens idéal du terme) dans un guide fini, on doit connaître l'impédance terminale de ce mode.

3.3.3.3 La transition entre les deux bandes : $\varphi=0$. - $p$ et $u$ peuvent se mettre sous la forme, si $X=\frac{x}{l}$ :

$$
\left\{\begin{array}{l}
p=X \Delta\left(Z_{0}-\zeta\right) u_{0}+p_{0} \\
u=X \Delta\left(1 / \zeta-Y_{0}\right) p_{0}+u_{0}
\end{array}\right.
$$

Quant à $e$ :

$$
e=x^{2}|\tilde{U}|^{2}+2 x \operatorname{Re}\left(\widetilde{U} V^{*}\right)+e_{0}
$$

où

$$
\tilde{U}=(L A-M \Delta) u_{0}+(M D+L \Delta) p_{0} .
$$

Donc $e^{\prime \prime}$ est constante, positive ou nulle. 
Si $e^{\prime \prime}$ est nulle, $e^{\prime}$ aussi et $e$ est constante. C'est en particulier le cas si $Z_{0}=\zeta$, c'est-à-dire dans le cas idéal.

Si $e^{\prime \prime}$ est positive, tout ce que nous avons dit pour la bande d'arrêt idéale reste valable. Il est alors à noter que si les fonctions $A\left(\varphi^{2}\right), B\left(\varphi^{2}\right), C\left(\varphi^{2}\right), D\left(\varphi^{2}\right)$ et $Z_{0}\left(\varphi^{2}\right)$ sont continues, $e^{\prime \prime}\left(\varphi^{2}\right)$ aussi et il existe donc un domaine de la bande passante idéale où $e^{\prime \prime}$ est partout positive.

3.3.3.4 Conclusion. - Dans les cas pratiques, il n'y a pas de séparation nette entre les bandes passante et d'arrêt idéales : pour $\varphi^{2}$ assez grand, la fonction amplitude $e(x)$ présente plusieurs arches de sinusoïdes, puis quand $\varphi^{2}$ décroît vers zéro, la concavité de la courbe $e(x)$ tend à être partout positive ou nulle. Quand $\varphi^{2}$ devient négatif, la concavité est toujours positive; sous certaine condition, la courbe est de plus monotone décroissante, ce qui constitue une généralisation partielle du cas idéal. Cette condition porte sur l'impédance de sortie; pour une ligne symétrique, il suffit que $j / \zeta \operatorname{Im}\left(Y_{0}\right)$ soit négatif (où $\zeta$ est imaginaire pur).

La figure 4 montre l'exemple du pavillon acoustique exponentiel (traité au § 5.3). Dans le cas particulier choisi, il débouche sur un tuyau cylindrique fermé à son extrémité, et, dans un but de réalisme, comporte des pertes. La figure représente les variations de l'énergie totale le long du pavillon à différentes fréquences : on voit que peu en dessous de la fréquence de coupure idéale $f_{\mathrm{c}}$, on a bien des ondes décroissantes. Par contre, quand la fréquence devient plus basse, l'énergie totale demeure une fonction à concavité positive, mais devient croissante. (Nous traitons au paragraphe suivant le problème de la détermination physique des fréquences de coupure pour cet exemple.)

4. Etude de l'affaiblissement par une ligne. Discussion de la notion de fréquence de coupure. - 4.1 PosITION DU PROBLÈME. - Dans bien des cas pratiques, notamment pour les filtres, on s'intéresse avant tout au rapport d'amplitude entre l'entrée et la sortie de la ligne. Ce rapport est en effet directement accessible expérimentalement, et la connaissance de ses variations avec la constante de propagation permet de définir une valeur critique pratique de celle-ci, délimitant des bandes passante et d'arrêt qui peuvent être différentes des bandes passante et d'arrêt idéales.

L'exemple le plus courant est celui des filtres, pour lesquels la constante de propagation dépend de la fréquence, le problème étant alors la recherche d'une fréquence de coupure. Mais ce qui suit vaut aussi quand la constante de propagation dépend d'une autre grandeur que la fréquence, comme par exemple l'angle d'incidence pour la réflexion d'une onde oblique.

Nous voulons donc étudier les variations de la fonction amplitude $E\left(\varphi^{2}\right)=e_{x} / e_{0}\left(\varphi^{2}\right): E$ varie dans le même sens que l'affaiblissement; elle est l'inverse de ce qu'on appelle habituellement la réponse (On se
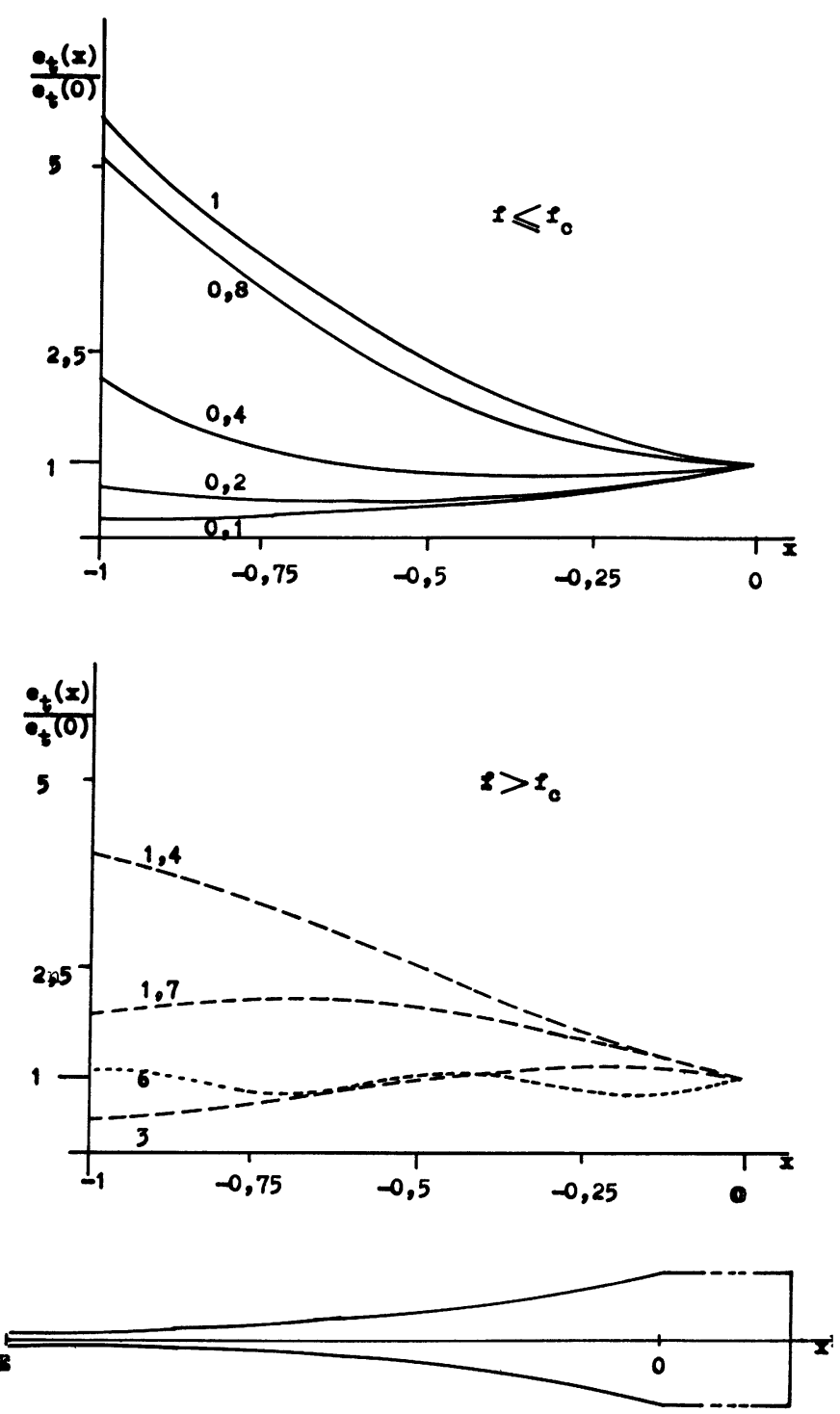

Fig. 4. - Variation de l'énergie totale le long d'un pavillon exponentiel (débouchant sur un cylindre fermé) pour diffé.rentes valeurs de $f / f_{\mathrm{c}}$.

[Variation of total energy along an exponential horn (terminated in a closed cylindrical tube) for several values of $f / f_{c}$.]

fixe une ligne de longueur donnée, la sortie étant en 0 , et l'entrée en $x$, qui est négatif).

4.2 Le CAS IDÉAL. - Dans le cas idéal $E=1$ dans la bande passante ( $\varphi^{2}$ positif), et dans la bande d'arrêt $\left(\varphi^{2}\right.$ négatif, $\left.j \varphi=\alpha\right), E=e^{-2 \alpha x}$, supérieur à 1 . La fonction $E\left(\varphi^{2}\right)$ a donc l'allure suivante, identique pour les trois formes d'énergie (Fig. 5).

La dérivée de $E$ par rapport à $\varphi^{2}$ présente donc une discontinuité pour $\varphi$ nul. Ceçi permet une définition précise de la valeur critique de $\varphi^{2}$, et donc de la fréquence de coupure : c'est la valeur de $\varphi^{2}$ au-dessous de laquelle il y a affaiblissement, et au-dessus de laquelle il n'y a pas affaiblissement. 


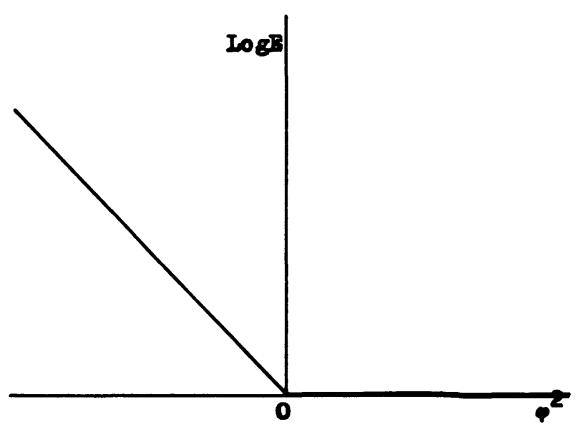

Fig. 5. - Variation de la fonction $\log E\left(\varphi^{2}\right)$ dans le cas idéal.

[Variation of function $\log E\left(\varphi^{2}\right)$ in the ideal case.]

4.3 DifFiculté DE LA GÉNÉRALISATION AUX CAS PRATIQUES. - Nous avons montré au $\S 3$ que en général, pour toute valeur de $\varphi^{2}, E$ pouvait être aussi bien supérieure qu'inférieure à 1 . Il est donc exclu dans le cas général de définir une fréquence de coupure, des bandes passantes et d'arrêt. La discontinuité de la dérivée de $E$ n'existe pas dans les cas pratiques, à cause de la réalisation imparfaite de l'impédance itérative (mais aussi à cause de l'existence de pertes). Ceci impose de choisir arbitrairement un critère pour définir une fréquence de coupure, quand cela est possible.

C'est ainsi que la définition des normes américaines est, pour un filtre passe-bande : "Les fréquences de coupure nominales supérieure et inférieure d'un filtre passe-bande sont les fréquences au-dessus et audessous de la fréquence de réponse maximale du filtre, auxquelles la réponse à un signal sinusoïdal est $3 \mathrm{~dB}$ au-dessous de la réponse maximale. »

Une telle définition ne peut bien entendu être employée que cas par cas. Elle est possible notamment dans de nombreux cas où la courbe $E\left(\varphi^{2}\right)$ est peu différente de celle du cas idéal : décroissante audessous d'une certaine valeur de $\varphi^{2}$, puis présentent de légères oscillations au-dessus. Cette forme de courbe n'est certes ni nécessaire ni suffisante pour permettre l'application de la définition ci-dessus, mais elle est souvent une indication intéressante. C'est pourquoi nous avons cherché à quelles conditions la courbe $E\left(\varphi^{2}\right)$ est décroissante dans la bande d'arrêt idéale.

4.4 ETUde DE LA FONCTION $E(\alpha)$ DANS LA BANDE D'ARRÊT IDÉALE. $-E$ peut se mettre sous la forme :

$$
E=\frac{\sinh ^{2} \alpha x}{2 \alpha^{2}} E_{0}^{\prime \prime}+\frac{\sinh 2 \alpha x}{2 \alpha} E_{0}^{\prime}+1
$$

On suppose que la condition $E_{0}^{\prime}$ négative ou nulle, trouvée au § 3.3.4 est satisfaite (' et " représentent les dérivées par rapport à $x$ ). De plus, on sait que $E_{0}^{\prime \prime}$ est positive.
On cherche à quelle condition $E$ croît avec $\alpha$ c'est-à-dire décroît avec $\varphi^{2}$, comme dans le cas idéal. Cette condition dépend en général de $x$; aussi nous chercherons simplement une condition nécessaire et suffisante pour que $\partial E / \partial \alpha$ soit positive quels que soient $x$ (négatif), et $\alpha$ (positif).

On sait que les fonctions $\frac{\sinh ^{2} \alpha x}{2 \alpha^{2}}$ et $-\frac{\sinh 2 \alpha x}{2 \alpha}$ sont positives et croissantes avec $\alpha$. Une condition suffisante est immédiate : $\frac{\partial E_{0}^{\prime \prime}}{\partial \alpha} \geqslant 0$ et $\frac{\partial E_{0}^{\prime}}{\partial \alpha} \leqslant 0$. Elle est de plus nécessaire, car si $x$ et $\alpha$ sont suffisamment petits, $E=1+x E_{0}^{\prime}+\frac{1}{2} x^{2} E_{0}^{\prime \prime} ;$ si $\frac{\partial E_{0}^{\prime}}{\partial \alpha}$ était positif, ou si $\frac{\partial E_{0}^{\prime \prime}}{\partial \alpha}$ était négatif, il existerait $x$ tel que $\frac{\partial E}{\partial \alpha}$ fût négatif.

Par conséquent, on a une généralisation du cas idéal si : $\frac{\partial E_{0}^{\prime}}{\partial \alpha} \leqslant 0$ et $\frac{\partial E_{0}^{\prime \prime}}{\partial \alpha} \geqslant 0$.

Nous en donnerons un exemple au $\S 5.3$.

Remarque : on peut s'intéresser aussi à $e_{x}(\alpha)$. Cette fonction vérifie :

$$
e_{x}=\frac{\sinh ^{2} \alpha x}{2 \alpha^{2}}+\frac{\sinh 2 \alpha x}{2 \alpha} e_{0}^{\prime}+e_{0} .
$$

Un raisonnement analogue au précédent donnerait la même condition nécessaire et suffisante, à laquelle il faut seulement ajouter la condition $\partial E_{0} / \partial \alpha$ positive.

4.5 Conclusion. - Dans les cas pratiques, on est obligé d'adopter un critère arbitraire pour définir une fréquence de coupure. De plus, il n'est pas possible de le faire pour toute ligne.

Ceci est bien connu; mais nous avons voulu montrer que la fréquence de coupure est une notion globale, et non locale : elle est définie pour une ligne de longueur donnée, et d'impédance terminale donnée. Ceci est important pour les lignes à caractéristiques variables (dont les caractéristiques varient avec $x$ ). Pour ce type de ligne, certains [36, 37] définissent une fréquence de coupure point par point, comme la fréquence de coupure que présenterait une ligne infinie dont les caractéristiques seraient celles de ce point; mais une telle définition est à notre avis assez abstraite. Nous en donnons un exemple avec les pavillons acoustiques $(\$ 6.4)$.

L'exemple montré par la figure 6 est le même que celui de la figure 4 . On voit que seule l'énergie cinétique pourrait permettre la définition d'une fréquence de coupure proche de la fréquence de coupure idéale $f_{c}$. Au-dessous de $f_{\mathfrak{c}}$, l'énergie potentielle est renforcée et non atténuée, et il en est de même pour l'énergie totale pour les fréquences inférieures à environ $0,3 f_{c}$. Nous montrerons au $\S 5.3$ que les conditions trouvées au $\S 4.4$ ne sont remplies effectivement que pour l'énergie cinétique. 

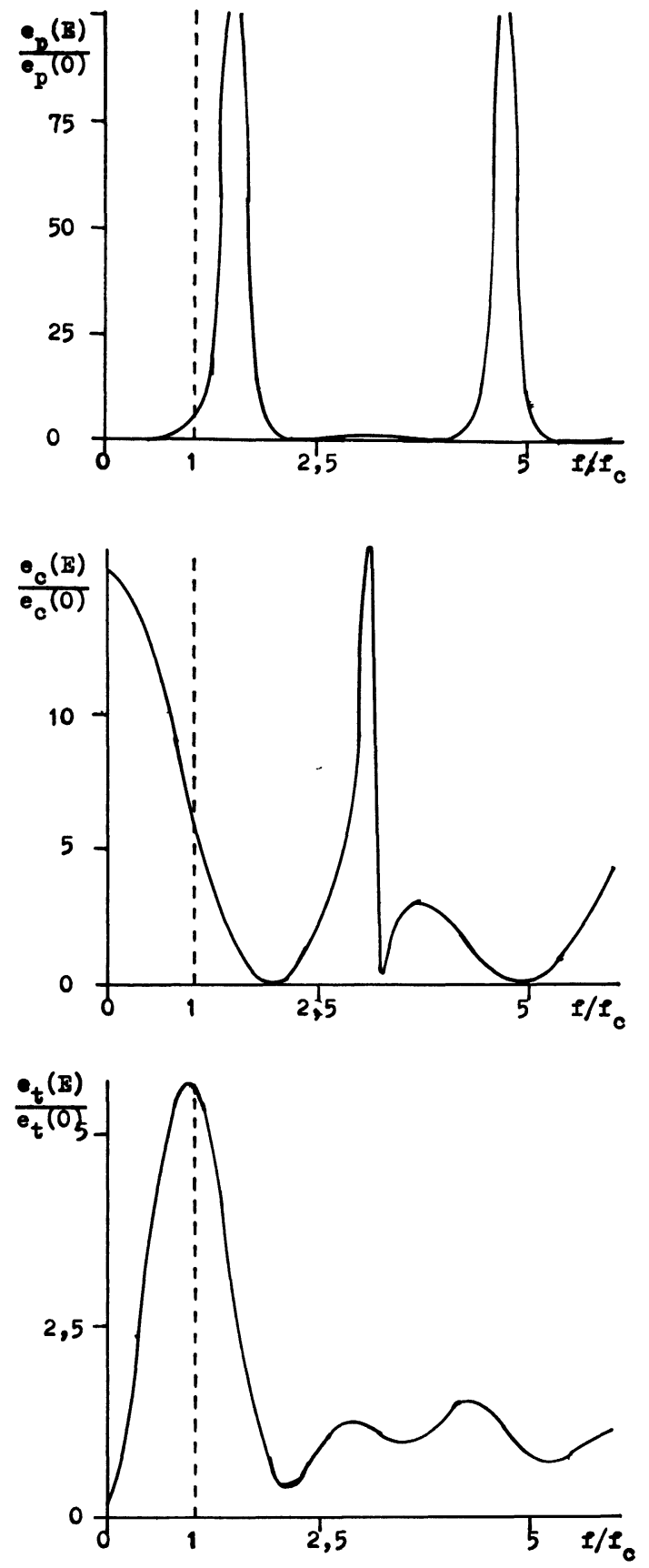

Fig. 6. - Variations des énergies potentielle, cinétique et totale en fonction de la fréquence pour un pavillon exponentiel (cf. Fig. 5).

[Variations of potential energy, kinetic energy and total energy as function of frequency for an exponential horn (cf. Fig. 5).]

5. Exemples de lignes sans pertes d'impédance terminale quelconque. - Les trois exemples qui suivent sont tirés de l'acoustique; mais ils ont évidemment leurs analogues en électromagnétisme. Pour tous, nous chercherons la condition à vérifier pour que dans la bande d'arrêt idéale, les ondes soient décroissantes (cf. $\S 3$ ) ; de plus, pour le dernier, nous discuterons l'existence de fréquences de coupure.
5.1 EXEMPLE DE LIGNE SYMÉTRIQUE : UNE ONDE RÉFRACTÉE À L'INTERFACE DE DEUX MILIEUX DIFFÉRENTS. - Nous voulons étudier ici le problème de la réfraction et de la réflexion d'une onde plane à l'interface (supposée infinie) de deux milieux 1 et 2 . Le milieu 2 étant supposé fini, quelle est la forme des ondes qui le traversent ? Soient $n_{1}, n_{1}^{\prime}, n_{2}, n_{2}^{\prime}$ les directions des ondes incidente, réfléchie, transmise et rétrograde (due à la présence du milieu 3) (Fig. 7).

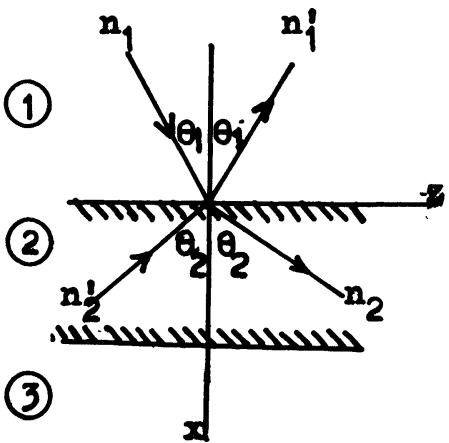

Fig. 7. - Onde oblique réfractée à l'interface de deux milieux 1 et 2 .

[Refraction of oblique wave at interface of two media 1 and 2.]

La pression s'écrit dans les milieux 1 et 2 :

$$
\begin{aligned}
& p_{1}=p_{\mathrm{i}}\left(\mathrm{e}^{-j k_{1} \cos \theta_{1} x}+R_{1} \mathrm{e}^{j k_{1} \cos \theta_{1} x}\right) \mathrm{e}^{-j k_{1} \sin \theta_{1} z} \mathrm{e}^{j \omega t} \\
& p_{2}=p_{\mathrm{i}} T\left(\mathrm{e}^{-j k_{2} \cos \theta_{2} x}+R_{2} \mathrm{e}^{j k_{2} \cos \theta_{2} x}\right) \mathrm{e}^{-j k_{2} \sin \theta_{2} z} \mathrm{e}^{j \omega t} .
\end{aligned}
$$

Quant à la composante $u_{x}$ de la vitesse dans le milieu 2 :

$$
\begin{aligned}
u_{x 2}=\frac{p_{\mathrm{i}} T \cos \theta_{2}}{\rho c}\left(\mathrm{e}^{-j k_{2} \cos \theta_{2} x}-R_{2}\right. & \left.\mathrm{e}^{j k_{2} \cos \theta_{2} x}\right) \times \\
& \times \mathrm{e}^{-j k_{2} \sin \theta_{2} z} \mathrm{e}^{j \omega t} .
\end{aligned}
$$

Chaque milieu peut être considéré comme une ligne (symétrique) pour la pression et la composante $u_{x}$ de la vitesse, $z$ étant fixé. Certes ces deux grandeurs ne caractérisent pas totalement l'onde, mais la grandeur supplémentaire, $u_{z}$, est constamment proportionnelle à $p: u_{z}=(\sin \theta / \rho c) p$. Tout ce que nous avons dit sur les lignes est donc applicable ici : le flux d'énergie moyen est le flux dans la direction $x$, et l'énergie totale est donnée par :

$$
\begin{aligned}
e & =\frac{1}{4 \rho c^{2}}|p|^{2}+\frac{1}{4} \rho\left(\left|u_{x}\right|^{2}+|u z|^{2}\right) \\
& =\frac{1}{4 \rho c^{2}}\left(1+\sin ^{2} \theta\right)|p|^{2}+\frac{1}{4} \rho\left|u_{x}\right|^{2} .
\end{aligned}
$$

(La ligne étant symétrique, le facteur placé devant $|p|^{2}$ n'a de toute façon pas d'influence sur le sens de variation de $e$.)

La loi de Descartes-Snell indique que

$$
\frac{\sin \theta_{1}}{c_{1}}=\frac{\sin \theta_{2}}{c_{2}} \text {. }
$$


Par conséquent, $\frac{1}{2} \pi-\theta_{2}$ est soit réel, soit imaginaire pur.

$\mathrm{Si} \frac{1}{2} \pi-\theta_{2}$ est réel, l'énergie totale dans le milieu 2 est une fonction périodique de $x$ (cf. 3.3.3.1). Elle est constante si le milieu 2 est infini.

Si $\frac{1}{2} \pi-\theta_{2}$ est imaginaire pur, ce qui correspond à la bande d'arrêt idéale, l'énergie est une fonction décroissante à condition que $\operatorname{Im}\left(Y_{x}\right)$ soit négative sur le plan de séparation du milieu 2. D'une façon générale, l'amplitude des ondes est alors décroissante, et nous avons donc partiellement généralisé le cas où le milieu 2 est infini.

Un cas particulier intéressant est le cas où le milieu 3 sur lequel débouche le milieu 2 est infini. Les lois de Descartes-Snell donnent :

$$
\frac{\sin \theta_{1}}{c_{1}}=\frac{\sin \theta_{2}}{c_{2}}=\frac{\sin \theta_{3}}{c_{3}} \text {. }
$$

Deux cas peuvent se présenter :

- Soit $\frac{1}{2} \pi-\theta_{3}$ est imaginaire pur (il y aurait réflexion totale s'il n'y avait pas de milieu 2). On a alors des ondes évanescentes dans le milieu 3 :

$$
p=a \mathrm{e}^{-k_{3} \sinh \chi x_{e}-j k_{3} \sin \theta_{3} z} \mathrm{e}^{j \omega t},
$$

où $\theta_{3}=\frac{1}{2} \pi+j \chi(\chi$ positif $)$.

L'admittance à la frontière 2-3 est $Y_{x}=\frac{\sinh \chi}{j \rho_{3} c_{3}}$.

Par conséquent $\operatorname{Im}\left(Y_{x}\right)$ est négative. Dans ce cas, on a donc des ondes décroissantes dans le milieu 2 (de manière d'ailleurs non exponentielle, bien que le flux d'énergie soit nul).

- Soit $\frac{1}{2} \pi-\theta_{3}$ est réel (il n'y aurait pas réflexion totale s'il n'y avait pas de milieu 2). On a alors des ondes propagatives dans le milieu 3 : L'admittance à la frontière 2-3 est :

$$
Y_{x}=\frac{\cos \theta_{3}}{\rho_{3} c_{3}}, \text { et donc } \operatorname{Im}\left(Y_{x}\right)=0 .
$$

On a encore des ondes décroissantes dans le milieu 2, mais la dérivée de l'amplitude de l'énergie s'annule à la frontière 2-3. Nous avons donc précisé la forme des ondes de « fuite » dont parle Brekhovskikh à propos d'une couche finie.

Ce dernier cas est en particulier celui d'une couche finie dans un milieu homogène (milieu 3 identique au milieu 1), traité par de nombreux auteurs (Rayleigh [38], Fiorito et Uberall [39]).

5.2 EXEMPLE DE LIGNE SYMÉTRIQUE : UN MODE NON PLAN DANS UN GUIDE FINI À PAROIS PARFAITEMENT RÉFLÉCHISSANTES. - Ce problème est identique au précédent, comme nous allons le montrer. Pour simplifier, nous choisirons un guide fini à deux dimensions, c'est-à-dire infini dans une direction (Fig. 8) :

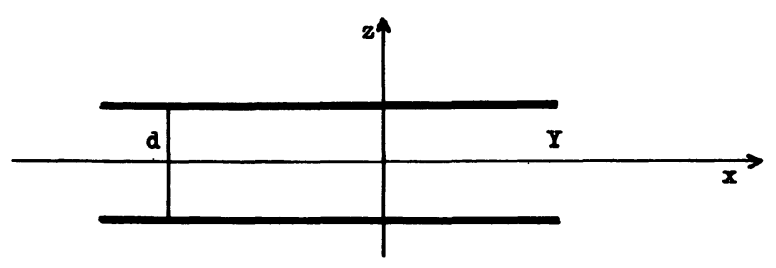

Fig. 8. - Guide d'onde fini.

[Finite waveguide.]

Les parois étant parfaitement réfléchissantes, la solution pour la pression et les composantes $u_{z}$ et $u_{x}$ s'écrit classiquement :

$$
\begin{aligned}
p & =\left(a \mathrm{e}^{-j k \cos \theta x}+b \mathrm{e}^{j k \cos \theta x}\right) \cos (k \sin \theta z) \mathrm{e}^{j \omega t} \\
u_{z} & =\frac{\sin \theta}{j \rho c}\left(a \mathrm{e}^{-j k \cos \theta x}+b \mathrm{e}^{j k \cos \theta x}\right) \sin (k \sin \theta z) \mathrm{e}^{j \omega t} \\
u_{x} & =\frac{\cos \theta}{\rho c}\left(a \mathrm{e}^{-j k \cos \theta x}-b \mathrm{e}^{j k \cos \theta x}\right) \cos (k \sin \theta z) \mathrm{e}^{j \omega t}
\end{aligned}
$$

où $k \sin \theta=\frac{m \pi}{a}$, avec $m=0,1,2,3 \ldots$

On ne retiendra que les valeurs de $m$ non nulles, c'est-à-dire les modes non plans. Pour ceux-ci, on définit classiquement une fréquence de coupure quand le guide est infini : elle est obtenue pour $k_{\mathrm{c}}=\frac{m \pi}{a}$.

Les raisonnements tenus au $\S 5.1$ restent applicables ici, à une seule modification près : il faut prendre les densités d'énergie par tranche $(x=$ constante) pour pouvoir considérer les modes orthogonaux isolément. Dans ces conditions, comme

$\frac{1}{a} \int_{0}^{a} \cos ^{2}(k \sin \theta z) \mathrm{d} z=\frac{1}{a} \int_{0}^{a} \sin ^{2}(k \sin \theta z) \mathrm{d} z=\frac{1}{2}$,

on obtient encore $\left|u_{z}\right|^{2}=|p|^{2} \sin ^{2} \theta$ d'où

$$
e=\frac{1}{2}\left(\frac{1}{4} \frac{1}{\rho c^{2}}\left(1+\sin ^{2} \theta\right)|p|^{2}+\frac{1}{4} \rho\left|u_{x}\right|^{2}\right) .
$$

Au-dessus de la fréquence de coupure idéale, $k \cos \theta$ est réel, et l'énergie moyenne par tranche oscille autour d'une valeur moyenne. Au contraire au-dessous, $k \cos \theta$ est imaginaire pur, et on peut avoir des ondes décroissantes pour la même condition qu'au paragraphe précédent : il faut qu'à l'extrémité du guide, on ait $\operatorname{Im}\left(Y_{x}\right)<0$.

5.3 EXEMPLE DE LIGNE ASYMÉTRIQUE : LE PAVILLON EXPONENTIEL DE SECTION $S=S_{0} \exp (2 m x)$. Comme on sait, l'équation des pavillons, dite de Webster, est une approximation, d'ailleurs bien vérifiée par l'expérience pour les basses fréquences. La conservation de l'énergie s'écrit, si $p$ et $u$ sont la pression et la vitesse acoustiques, et $S$ la section :

$$
\frac{1}{2} S \operatorname{Re}\left(p u^{*}\right)=\text { constante . }
$$


Autrement dit, il est nécessaire de rapporter la pression et la vitesse à la section, pour considérer le pavillon comme une ligne sans pertes : les grandeurs caractérisant la ligne sont donc $p d$ et $u d$, où $d$ est le diamètre du pavillon. On a alors :

$$
\begin{aligned}
& p d=\left(a \mathrm{e}^{-\alpha x}+b \mathrm{e}^{\alpha x}\right) \mathrm{e}^{j \omega t} \\
& u d=\left(\frac{m}{j k} \frac{1}{\rho c}\left(a \mathrm{e}^{-\alpha x}+b \mathrm{e}^{\alpha x}\right)+\right. \\
&\left.\quad+\frac{\alpha}{j k} \frac{1}{\rho c}\left(a \mathrm{e}^{-\alpha x}-b \mathrm{e}^{\alpha x}\right)\right) \mathrm{e}^{j \omega t}
\end{aligned}
$$

avec $\alpha^{2}=m^{2}-k^{2}$

L'énergie totale par tranche est alors :

$$
e=\frac{S}{4}\left(\frac{|p|^{2}}{\rho c^{2}}+\rho|u|^{2}\right)
$$

Le pavillon exponentiel est donc une ligne asymétrique; les conditions pour avoir des ondes décroissantes au-dessous de la fréquence de coupure idéale $f_{\mathrm{c}}$ sont :

$$
\left|Y_{0}\right|>\frac{1}{\rho c}
$$

$$
\text { pour l'énergie totale par }
$$
tranche.
$\operatorname{Im}\left(Y_{0}\right)<-\frac{1}{\rho c} \frac{m}{k} \underset{\text { par tranche. }}{\text { pour l'énergie potentielle }}$

$\operatorname{Im}\left(Z_{0}\right)<\frac{m}{k} \rho c$ pour l'énergie cinétique par tranche.

(On vérifie bien que les deux dernières conditions impliquent la première.) Nous voulons montrer que l'impédance de rayonnement vérifie effectivement ces conditions :

En général, le produit $\left(k r_{0}\right)$, où $r_{0}$ est le rayon de sortie, est très inférieur à 1 pour $f_{\mathrm{c}}$; il est en effet égal à $m r_{0}$, et les hypothèses de l'équation des pavillons imposent $m$ et $r_{0}$ petits. Dans ces conditions, quelle que soit la formule choisie pour l'impédance de rayonnement (par exemple Rayleigh) :

$$
Z_{0}=\rho c\left(\frac{1}{2}\left(k r_{0}\right)^{2}+j \frac{8 \pi}{3}\left(k r_{0}\right)\right),
$$

celle-ci est en module trés inférieure à $\rho c$ pour $f<f_{\text {c }}$. Par conséquent, la condition des ondes décroissantes est vérifiée pour l'énergie totale. On montre immédiatement qu'il en est de même pour les énergies potentielle et cinétique, $\operatorname{car} m r_{0}<\frac{3 \pi}{8}$.

A propos de l'existence de fréquences de coupure (cf. §4.3), on obtient les conditions suivantes :

\begin{tabular}{|c|c|c|}
\hline Energie & $\frac{\partial E_{0}^{\prime}}{\partial \alpha}<0$ & $\frac{\partial E_{0}^{\prime \prime}}{\partial \alpha}>0$ \\
\hline$E_{\mathrm{p}}=\frac{e_{\mathrm{p}(x)}}{e_{\mathrm{p}(0)}}$ & $\frac{\partial}{\partial k}\left(k \operatorname{Im}\left(Y_{0}\right)\right)>0$ & $\frac{\partial}{\partial k}\left(\left|Y_{0}\right|^{2} k^{2} \rho^{2} c^{2}-k^{2}+2 m k \rho c \operatorname{Im}\left(Y_{0}\right)\right)<0$ \\
\hline$E_{\mathrm{c}}=\frac{e_{\mathrm{c}(x)}}{e_{\mathrm{c}(0)}}$ & $\frac{\partial}{\partial k}\left(k \operatorname{Im}\left(Z_{0}\right)\right)>0$ & $\frac{\partial}{\partial}\left(\frac{\left|Z_{0}\right|^{2}}{\rho^{2} c^{2}} k^{2}-k^{2}-2 \frac{m k}{\rho c} \operatorname{Im}\left(Z_{0}\right)\right)<0$ \\
\hline$E_{\mathrm{t}}=\frac{e_{\mathrm{t}(x)}}{e_{\mathrm{t}(0)}}$ & $\frac{\partial}{\partial k}\left(\left|Y_{0}\right|^{2}\right)<0$ & $\frac{\partial}{\partial k}\left(\frac{k \operatorname{Im}\left(Y_{0}\right)}{1+\left|Y_{0}\right|^{2} \rho^{2} c^{2}}\right)<0$ \\
\hline
\end{tabular}

On peut vérifier que toutes ces conditions sont bien satisfaites par l'impédance de rayonnement aux basses fréquences. (Le calcul est rapide si on ne conserve que les termes de plus bas degré en $k r_{0}$.) On peut par conséquent affirmer qu'un pavillon rayonnant dans l'espace infini transmet effectivement très peu d'énergie au-dessous de sa fréquence de coupure idéale. Ceci justifie le point de vue d'Olson (cité au $\S 3.3 .1$ ), et l'assimilation courante faite entre la fréquence de coupure d'un tel pavillon fini et celle d'un pavillon infini.

Discussion de l'exemple des figures 4 et 6. - Le pavillon choisi a les dimensions suivantes : longueur $1 \mathrm{~m}, r_{0}=0,1 \mathrm{~m} ; m=1$; il débouche sur un tuyau cylindrique fermé de longueur $l=1 \mathrm{~m}$, et de rayon $0,1 \mathrm{~m}$.
Les pertes sont les pertes visco-thermiques aux parois ; le calcul est numérique, et est bien confirmé par l'expérience pour de nombreux tuyaux (cf. notamment [40]). On peut admettre que, sauf aux résonances, les pertes jouent un rôle sans importance qualitative. C'est pourquoi nous allons chercher si les conditions des $\S 3$ et $\mathbf{4}$ sont remplies en négligeant les pertes.

Le tuyau cylindrique étant fermé, son admittance d'entrée, qui est l'admittance de sortie $Y_{0}$ du pavillon exponentiel, s'écrit : $Y_{0}=\frac{1}{\rho c} j \operatorname{tg} k l$. Comme $l=1 \mathrm{~m}$, et le nombre d'onde de coupure idéal $k_{\mathrm{c}}=m=1$, on a : si $k<k_{\mathrm{c}}, 0<k l<1<\frac{1}{2} \pi$, d'où tg $k l>0$.

Par conséquent, la condition pour avoir des ondes décroissantes est vérifiée pour l'énergie cinétique quel 
que soit $k<k_{\mathrm{c}}$, et jamais pour l'énergie potentielle. Pour l'énergie totale, elle est vérifiée si $\operatorname{tg} k l>1$, c'est-à-dire si $k>\frac{\pi}{4} k_{\mathrm{c}}$. C'est ce que montre la figure 4 .

Quant aux conditions relatives à l'existence des fréquences de coupure, elles ne sont vérifiées que pour l'énergie cinétique, ce qui confirme bien les résultats présentés par la figure 6 .

6. Les lignes sans pertes à caractéristiques variables. - 6.1 Notations UTILISÉES. - Nous voulons ici généràliser le $\S 3$ pour les lignes à caractéristiques variables; nous partirons du système différentiel 7 (§ 2.6) :

$$
\left\{\begin{aligned}
p & =(\bar{\mu}+\bar{\gamma}) u+\bar{\beta} u^{\prime} \\
u & =(\mu+\gamma) p+\beta p^{\prime}
\end{aligned}\right.
$$

où $\mu$ et $\bar{\mu}$ sont réels, $\gamma$ et $\bar{\gamma}$ imaginaires purs, $\beta$ et $\bar{\beta}$ complexes, tous étant désormais des fonctions de $x$.

On suppose de plus le flux d'énergie constant : $\operatorname{Re}\left(p u^{*}\right)^{\prime}=0 \Leftrightarrow \beta$ et $\bar{\beta}$ imaginaires purs,

$$
\frac{\gamma}{\beta}=-\frac{\bar{\gamma}}{\bar{\beta}} \text { et } \frac{\mu}{\beta}=\frac{\bar{\mu}}{\bar{\beta}} \text {. }
$$

Si on pose $v=\exp \left(\int \frac{\mu}{\beta} \mathrm{d} x\right)$ (où $v$ est de module 1 , puisque $\mu / \beta$ est imaginaire pur), on peut éliminer $\mu$ et $\bar{\mu}$ du système différentiel :

$$
\left\{\begin{array}{l}
(p v)=\bar{\gamma}(u v)+\bar{\beta}(u v)^{\prime} \\
(u v)=\gamma(p v)+\beta(p v)^{\prime} .
\end{array}\right.
$$

La condition $\mu=\bar{\mu}=0$ est donc équivalente à la condition $W=0$ (cf. $\S 3.1$ ); nous la supposerons remplie dans ce qui suit. Nous étudierons en définitive le système suivant $(v=1)$ :

$$
\left.\begin{array}{l}
p=\bar{\gamma} u+\bar{\beta} u^{\prime} \\
u=\gamma p+\beta p^{\prime}
\end{array}\right\}
$$

où $\beta, \bar{\beta}, \gamma, \bar{\gamma}$ sont imaginaires purs et $\frac{\gamma}{\beta}=-\frac{\bar{\gamma}}{\bar{\beta}}$.

6.2 ETUDE DE LA VARIATION D'AMPLITUDE DE $e(x)=L^{2}|p|^{2}+M^{2}|u|^{2}$ LE LONG DE LA LIGNE. Le système différentiel implique les deux équations suivantes :

$$
\begin{aligned}
u^{\prime \prime}+u^{\prime} \frac{\bar{\beta}^{\prime}}{\bar{\beta}}+u\left(\varphi^{2}+\frac{\overline{\gamma^{\prime}}}{\bar{\beta}}\right)=0 \\
p^{\prime \prime}+p^{\prime} \frac{\beta^{\prime}}{\beta}+p\left(\varphi^{2}+\frac{\gamma^{\prime}}{\beta}\right)=0
\end{aligned}
$$

où $\varphi^{2}=\frac{\bar{\gamma}-1}{\beta \bar{\beta}} ; \varphi^{2}, \frac{\beta^{\prime}}{\beta}, \frac{\bar{\beta}^{\prime}}{\bar{\beta}}, \frac{\gamma^{\prime}}{\beta}=-\frac{\overline{\gamma^{\prime}}}{\bar{\beta}}$

sont réels.

Le calcul de l'équation différentielle satisfaite par la fonction amplitude $e(x)$ est fastidieux ; nous donnons en annexe II la démonstration du résultat suivant, qui généralise celui du 3.3.3.2 :

$e(x)$ est décroissante pour tout $x$ négatif à condition que :

$$
e_{0}^{\prime}<0 \quad \text { et } \varphi^{2}<s \psi^{\prime}-\frac{1}{4} \psi^{\prime 2}
$$

où

$$
s=-\frac{1}{2} j\left(\frac{L}{M \beta}+\frac{M}{L \bar{\beta}}\right)
$$

et

$$
\psi=-\operatorname{arctg} \frac{1}{2} j\left(\frac{L}{M \gamma}+\frac{M}{L \bar{\gamma}}\right) .
$$

Si on fait tendre $L$ (ou $M$ ) vers zéro, on trouve les conditions suivantes, qui apparaissent d'ailleurs clairement dans le système (9) :

$$
\begin{aligned}
& -\operatorname{Si} L=0: \varphi^{2}<-\frac{\bar{\gamma}^{\prime}}{\bar{\beta}}=\frac{\gamma^{\prime}}{\beta} . \\
& \text { - Si } M=0: \varphi^{2}<-\frac{\gamma^{\prime}}{\beta} .
\end{aligned}
$$

On peut constater que les dérivées de $\beta$ et $\bar{\beta}$ n'interviennent pas dans ces résultats. Il s'en suit que pour une ligne symétrique $(\gamma=\bar{\gamma}=0)$ la condition se résume à $\varphi^{2}$ négatif, quels que soient $L$ et $M$, que les caractéristiques varient ou non.

On peut d'autre part remarquer que si $\frac{L^{2}}{\gamma}+\frac{M^{2}}{\gamma}$ est constant, la condition s'écrit aussi $\varphi^{2}$ négatif. C'est le cas en particulier de certaines lignes telles que $L^{2} \bar{\gamma}=-M^{2} \gamma$, c'est-à-dire telles que $L^{2} \bar{\beta}=M^{2} \beta$, ou encore avec les notations du $\S 2.1, L^{2} A=M^{2} D$. Cette relation porte sur les éléments $A$ et $D$ de la matrice du quadripôle; or un quadripôle tel que $B=C$ est appelé symétrique. C'est pourquoi nous proposons d'appeler les lignes vérifiant les relations précédentes lignes pseudo-symétriques, avec la définition suivante : lignes telles que l'énergie totale est proportionnelle à $D|p|^{2}+A|u|^{2}$, ou encore à $\beta|p|^{2}+\bar{\beta}|u|^{2}$.

Il nous reste à calculer $e^{\prime}$, avec les notations du système $(8)$ :

$$
\begin{aligned}
e^{\prime}=-2 \frac{\gamma}{\beta}\left(L^{2}|p|^{2}-\right. & \left.M^{2}|u|^{2}\right)+ \\
& +2 j\left(\frac{L^{2}}{\beta}-\frac{M^{2}}{\bar{\beta}}\right) \cdot \operatorname{Im}\left(p^{*} u\right) .
\end{aligned}
$$

C'est le même résultat que celui du §3.3.3.2; mais ici $\beta, \bar{\beta}$, et $\gamma$ varient. On peut remarquer que l'expression de $e^{\prime}$ est la somme d'un terme pseudo-symétrique et d'un terme symétrique.

6.3 Conclusion. - Si nous appelons $\left(s \psi^{\prime}-\frac{1}{4} \psi^{\prime 2}\right)$ (x) valeur critique de $\varphi^{2}$, nous pouvons dire : si $\varphi^{2}$ est partout inférieure à la valeur critique, et si $e_{0}^{\prime}$ est néga- 
tive, les ondes sont décroissantes, c'est-à-dire que l'énergie moyenne (potentielle, cinétique, ou totale selon les cas) est une fonction décroissante de $x$, bien que le flux d'énergie soit en général non nul.

Mais comme nous l'avons remarqué pour les caractéristiques constantes $(\S 3.3 .3 .4)$, l'énergie peut rester partout décroissante si $\varphi^{2}$ est supérieure à la valeur critique sur une fraction de longueur de la ligne, et même éventuellement sur toute la longueur.

La valeur critique de $\varphi^{2}$ est en général différente pour $p$ et $u$, sauf si la ligne est symétrique. Ceci justifie l'intérêt porté à l'énergie totale, qui est en quelque sorte un moyen terme, notamment pour les lignes pseudo-symétriques : pour ces dernières, la valeur critique correspondant à l'énergie totale est nulle, et elle est la moyenne des valeurs critiques correspondant aux énergies potentielle et cinétique.

Nous avons donc généralisé le $\S 3$ aux lignes à caractéristiques variables; on pourrait en faire autant du $\S 4$, c'est-à-dire étudier les variations en un point de $e$ avec $\varphi^{2}$. Afin de ne pas alourdir l'exposé, nous nous contenterons de noter que les conclusions sont les mêmes, que les caractéristiques varient ou non.

6.4 EXEMPLE DE LIGNES À CARACTÉRISTIQUES VARIABles : LeS PAVILlons ACOUSTIQUES. - Comme pour le pavillon exponentiel ( $\$ 5.3$ ), nous rapportons la pression $p$ et la vitesse $u$ à la section. On a alors :

$$
\begin{array}{r}
\beta=\frac{j}{k \rho c}, \quad \bar{\beta}=\frac{j}{k} \rho c, \quad \frac{\gamma}{\beta}=-\frac{d^{\prime}}{d}=-\frac{\bar{\gamma}}{\bar{\beta}}, \\
\varphi^{2}=k^{2}-\left(\frac{d^{\prime}}{d}\right)^{2}
\end{array}
$$

où $d(x)$ est le diamètre, $k$ le nombre d'onde, $\rho c$ l'impédance itérative des ondes planes. Le système (9) devient :

$$
\left\{\begin{array}{c}
(u d)^{\prime \prime}+(u d)\left(\varphi^{2}+\left(\frac{d^{\prime}}{d}\right)^{\prime}\right)=0 \\
(p d)^{\prime \prime}+(p d)\left(\varphi^{2}-\left(\frac{d^{\prime}}{d}\right)^{\prime}\right)=0
\end{array}\right.
$$

L'énergie totale s'écrivant

$$
e=\frac{S}{4}\left(\left|\frac{p}{\rho c^{2}}\right|^{2}+\rho|u|^{2}\right),
$$

la ligne est donc pseudo-symétrique. La valeur critique de $\varphi^{2}$ correspondant à l'énergie totale est donc nulle, et celles correspondant aux énergies potentielle et cinétique sont respectivement $\left(d^{\prime} / d\right)^{\prime}$ et $-\left(d^{\prime} / d\right)^{\prime}$.
Or quand il est possible de définir des fréquences de coupure (cf. $\S 4)$, on doit avoir :

$$
\begin{array}{cl}
f<f_{\mathrm{c}} \Rightarrow \varphi^{2}<\text { valeur critique } \forall x \\
\text { soit } f<f_{\mathrm{c}} \Rightarrow k^{2}<\inf \left(\text { valeur critique }+\left(d^{\prime} / d\right)^{2}\right) .
\end{array}
$$

On doit donc avoir nécessairement, pour les nombres d'onde de coupure $k_{\mathrm{c}}$ :

- pour l'énergie totale : $k_{\mathrm{c}}>\inf \left(d^{\prime} / d\right)$;

- pour l'énergie potentielle :

$$
k_{\mathrm{c}}>\inf \left(\left(d^{\prime} / d\right)^{2}+\left(d^{\prime} / d\right)^{\prime}\right)^{1 / 2} ;
$$

- pour l'énergie cinétique :

$$
k_{\mathrm{c}}>\inf \left(\left(d^{\prime} / d\right)^{2}-\left(d^{\prime} / d\right)^{\prime}\right)^{1 / 2} .
$$

On peut remarquer que l'équation des ondes sphériques est un cas particulier de l'équation des pavillons : il suffit de remplacer $x$ par $r$, rayon de la sphère, et $d^{\prime} / d$ par $1 / r$. Les inégalités ci-dessus deviennent alors : $k_{\mathrm{c}}$ respectivement supérieur à inf $(1 / r)$, à 0 , et à $\inf (\sqrt{2} / r)$. Si les conditions aux limites le permettent, on peut par conséquent définir une fréquence de coupure au moins pour l'énergie totale et l'énergie cinétique. Ce résultat montre l'intérêt de ne pas raisonner sur la seule pression.

7. Conclusion. - L'existence de deux types d'ondes bien distincts (ondes propagatives et ondes évanescentes) dans une ligne est une propriété des lignes idéales, infinies et sans pertes. Pour certaines lignes assez courtes, une telle distinction peut être complètement impossible. En fait, elle est liée à l'existence d'une discontinuité présentée par la dérivée de la fonction affaiblissement par rapport à la constante de propagation; or cette discontinuité ne peut résulter que d'un passage à la limite, les fonctions caractérisant une ligne étant dans la pratique $a$ priori continues.

C'est pourquoi l'assimilation d'une ligne très longue à une ligne infinie demande des précautions, même si dans de nombreux cas pratiques elle n'est pas contredite par l'expérience : il est exact que pour une impédance terminale (fonction de la constante de propagation) donnée, plus la ligne est longue, plus elle se rapproche du cas idéal ; par contre, cette affirmation n'est pas correcte si elle ne tient pas compte de l'impédance terminale.

Remerciements. - Je remercie vivement MM. Bergassoli, Bernard, Bosquet, Bouix, Bruneau, Chaigne, Garault, Gatignol, Hugonin, Pério, Poirée et Siestrunck de leurs conseils et suggestions concernant tant la forme que le fond de ce travail. 


\section{Annexe I : calcul de la fonction $e=L^{2}\left|p^{2}\right|+M^{2}|u|^{2}$}

1. Bande d arrêt idéale : $\varphi$ imaginaire pur. - On pose alors $j \varphi=\alpha, j \phi=\alpha l$ d'où

$$
\begin{aligned}
\sin \varphi l & =-j \sinh \alpha l \quad \text { et } \quad \frac{\sin x \varphi}{\sin \varphi l}=\frac{\sinh x \alpha}{\sinh \alpha l} \\
\cos \varphi l & =\cosh \alpha l
\end{aligned}
$$

1.1 NotaTION LINÉAIRE.

$$
\begin{array}{lll}
p=a \mathrm{e}^{-x}+b \mathrm{e}^{+x} & \zeta=\frac{A}{-\sinh \alpha l-\Delta}=\frac{-\sinh \alpha l+\Delta}{D} \\
u=a / \zeta \mathrm{e}^{-\alpha x}-b / \zeta^{\prime} \mathrm{e}^{+\alpha x} & \text { avec } & \zeta^{\prime}=\frac{A}{-\sinh \alpha l+\Delta}=\frac{-\sinh \alpha l-\Delta}{D}
\end{array}
$$

(on a $A D=\sinh ^{2} \alpha l-\Delta^{2} ; \zeta \zeta^{*}$ réel)

$$
\begin{aligned}
& |p|^{2}=|a|^{2} \mathrm{e}^{-2 \alpha x}+|b|^{2} \mathrm{e}^{2 \alpha x}+2 \operatorname{Re}\left(a b^{*}\right) \\
& |u|^{2}=|a|^{2} /\left.\zeta\right|^{2} \mathrm{e}^{-2 \alpha x}+|b|^{2} /\left|\zeta^{\prime}\right|^{2} \mathrm{e}^{2 \alpha x}+2 \operatorname{Re}\left(a b / \zeta \zeta^{\prime *}\right) .
\end{aligned}
$$

Soient $L$ et $M$ réels, tels que $L^{2}+M^{2} \neq 0$ :

$$
e=|a|^{2}\left(L^{2}+M^{2} /|\zeta|^{2}\right) \mathrm{e}^{-2 \alpha x}+|b|^{2}\left(L^{2}+M^{2} /\left|\zeta^{\prime}\right|^{2}\right) \mathrm{e}^{2 \alpha x}+2 \operatorname{Re}\left(a b^{*}\right)\left(L^{2}+M^{2} / \zeta \zeta^{*}\right) .
$$

Les dérivées de $e$ s'écrivent:

$$
\begin{aligned}
& e^{\prime}=-2 \alpha\left(|a|^{2}\left(L^{2}+M^{2} /|\zeta|^{2}\right) \mathrm{e}^{-2 \alpha x}-|b|^{2}\left(L^{2}+M^{2} /\left|\zeta^{\prime}\right|^{2}\right) \mathrm{e}^{2 \alpha x}\right. \\
& e^{\prime \prime}=4 \alpha^{2}\left(|a|^{2}\left(L^{2}+M^{2} /|\zeta|^{2}\right) \mathrm{e}^{-2 \alpha x}+|b|^{2}\left(L^{2}+M^{2} /\left|\zeta^{\prime}\right|^{2}\right) \mathrm{e}^{2 \alpha x}\right) .
\end{aligned}
$$

Par conséquent, $e^{\prime \prime}$ est positive quel que soit $x$.

La condition $e_{0}^{\prime}$ négative s'écrit :

$$
|a|^{2}\left(L^{2}+M^{2} /|\zeta|^{2}\right)>|b|^{2}\left(L^{2}+M^{2} /\left|\zeta^{\prime}\right|^{2}\right) .
$$

En effet, nous avons choisi $\operatorname{Im}(\varphi)<0$, et donc $\alpha>0$.

1.2 Notation MATRICIELle.

$$
\begin{aligned}
& e=|L p+M u|^{2}-2 L M \operatorname{Re}\left(p u^{*}\right)=|L p+M u|^{2}-2 L M \operatorname{Re}\left(p_{0} u_{0}^{*}\right) \\
& e=\left|\left(A_{n} L+C_{n} M\right) u_{0}+\left(B_{n} L+D_{n} M\right) p_{0}\right|^{2}-2 L M \operatorname{Re}\left(p_{0} u_{0}^{*}\right) \\
& e=|\sinh \alpha x U+\cosh \alpha x V|^{2}-2 L M \operatorname{Re}\left(p_{0} u_{0}^{*}\right) \\
& e=\sinh ^{2} \alpha x\left(|U|^{2}+|V|^{2}\right)+\operatorname{Re}\left(U^{*} V\right) \sinh 2 \alpha x+e_{0}
\end{aligned}
$$

avec

$$
U=\frac{(L A-M \Delta) u_{0}+(M D+L \Delta) p_{0}}{\sinh \alpha l} \quad \text { et } \quad V=L p_{0}+M u_{0}
$$

On en tire $e_{0}^{\prime}: e_{0}^{\prime}=2 \alpha \operatorname{Re}\left(U^{*} V\right)$. Or $A$ et $D$ sont imaginaires purs, et $\Delta$ réel, d'où :

$$
e_{0}^{\prime}=\frac{2 \alpha}{\sinh \alpha l}\left(\left(L^{2}\left|p_{0}\right|^{2}-M^{2}\left|u_{0}\right|^{2}\right)+j\left(L^{2} A-M^{2} D\right) \operatorname{Im}\left(p_{0}^{*} u_{0}\right)\right) .
$$

D'autre part, $\gamma=-\Delta / A$ et $\delta=-\sinh \alpha l / A$. Donc

$$
e_{0}^{\prime}=2 \alpha\left(\gamma / \delta\left(L^{2}\left|p_{0}\right|^{2}-M^{2}\left|u_{0}\right|^{2}\right)+j\left(-L^{2} / \delta+M^{2} \delta\left(1-\gamma^{2} / \delta^{2}\right)\right) \operatorname{Im}\left(p_{0}^{*} u_{0}\right)\right) .
$$

1.3 CalCul de $E_{0}^{\prime}$ ET $E_{0}^{\prime \prime}$, OU $E=e / e_{0} .-E_{0}^{\prime}=e_{0}^{\prime} /\left(L^{2}+M^{2}\left|Y_{0}\right|^{2}\right)$. soit :

Si on remarque que $|U|^{2}+|V|^{2}=|U+V|^{2}-2 \operatorname{Re}\left(U^{*} V\right)$, on obtient : $e_{0}^{\prime \prime}=2 \alpha^{2}|U+V|^{2}-2 \alpha e_{0}^{\prime}$,

$$
E_{0}^{\prime \prime}=-\frac{2 \alpha^{2}}{\delta^{2}}\left(L^{2}-M^{2} / \zeta^{2}\right) \frac{\left|Y_{0}-1 / \zeta\right|^{2}}{L^{2}+M^{2}\left|Y_{0}\right|^{2}}-2 \alpha E_{0}^{\prime} .
$$


2. Bande passante idéale $: \varphi$ réel. -2.1 CALCUL DE $e$. - Avec la notation linéaire, on a :

$$
\begin{gathered}
\left\{\begin{array}{llc}
|p|^{2}=|a|^{2}+|b|^{2}+2|a b| \cos (2 \varphi x-\theta) & \text { où } & \theta=\arg \left(a b^{*}\right) \\
|u|^{2}=\left(|a|^{2}+|b|^{2}-2|a b| \cos \left(2 \varphi x-\theta^{\prime}\right)\right) /|\zeta|^{2} & \text { où } & \theta^{\prime}=\arg \left(a b^{*} / \zeta \zeta^{*}\right)
\end{array}\right. \\
e=\left(|a|^{2}+|b|^{2}\right)\left(L^{2}+M^{2} /|\zeta|^{2}\right)+2|a b|\left(L^{2} \cos (2 \varphi x-\theta)-M^{2} /|\zeta|^{2} \cos \left(2 \varphi x-\theta^{\prime}\right)\right)
\end{gathered}
$$

(en effet $\zeta=\zeta^{\prime *}$ ).

Avec la notation matricielle, le calcul est semblable à celui de la bande d'arrêt :

$$
e=\sin ^{2} x \varphi\left(|U|^{2}-|V|^{2}\right)+\operatorname{Re}\left(U^{*} V\right) \sin 2 x \varphi+e_{0}
$$

où

$$
U=\frac{(L A-M \Delta) u_{0}+(M D+L \Delta) p_{0}}{\sin \varphi l} \quad \text { et } \quad V=L p_{0}+M u_{0}
$$

2.2 Calcul des extremums de $|p|$ ET $|u|$.

$$
\begin{array}{llll}
|p|_{\max }^{2}=\left(|a|^{2}+|b|^{2}\right) & \text { pour } & 2 \varphi x-\theta=0(2 \pi) \\
|p|_{\min }^{2}=\left(|a|^{2}-|b|^{2}\right) & \text { pour } & 2 \varphi x-\theta=\pi(2 \pi) \\
|u|_{\max }^{2}=\left(|a|^{2}+|b|^{2}\right) /\left.\zeta\right|^{2} & \text { pour } & 2 \varphi x-\theta^{\prime}=\pi(2 \pi) \\
|u|_{\min }^{2}=\left(|a|^{2}-|b|^{2}\right) /\left.\zeta\right|^{2} & \text { pour } & 2 \varphi x-\theta^{\prime}=0(2 \pi) .
\end{array}
$$

La coïncidence entre maximums de $|p|$ et minimums de $|u|$ n'a lieu que si $\theta=\theta^{\prime}$, c'est-à-dire $\operatorname{siarg}\left(\zeta^{2}\right)=0$, donc $\zeta$ réel, autrement dit si la ligne est symétrique. L'annulation de $|p|$ ou de $|u|$ ne peut avoir lieu que si $|b|=|a|$.

3. Cas limite $: \varphi=0$. - Quand $\varphi \rightarrow 0, \frac{\sin \varphi x}{\sin \varphi l} \rightarrow \frac{x}{l}=X$. D'où :

$$
\begin{aligned}
& p=X A u_{0}+(1+X \Delta) p_{0}=X \Delta\left(Z_{0}-\zeta\right) u_{0}+p_{0} \\
& u=(1-X \Delta) u_{0}+X D p_{0}=X \Delta\left(1 / \zeta-Y_{0}\right) p_{0}+u_{0}
\end{aligned}
$$

avec $A D=-\Delta^{2}$ et $\zeta=-\zeta^{\prime}=-A / \Delta=\frac{\Delta}{D}$ (imaginaire pur).

Si $\tilde{U}=(L A-M \Delta) u_{0}+(M D+L \Delta) p_{0}=(L \sqrt{A}-j M \sqrt{D})\left(\sqrt{A} u_{0}+j \sqrt{D} p_{0}\right)$ et $V=L p_{0}+M u_{0}$, on peut écrire $e$ sous la forme :

$$
e=X^{2}|\tilde{U}|^{2}+2 X \operatorname{Re}\left(\tilde{U} V^{*}\right)+e_{0} .
$$

D'où :

$$
\begin{aligned}
e^{\prime} l & =2 X|\tilde{U}|^{2}+2 \operatorname{Re}\left(U V^{*}\right) \\
e^{\prime \prime} l^{2} & =2|\tilde{U}|^{2}: c^{\prime e s t} \text { une constante positive ou nulle. }
\end{aligned}
$$

Etude du cas où e" est nulle

$$
\begin{aligned}
e^{\prime \prime} & =0 \Leftrightarrow \tilde{U}=0 \Rightarrow e^{\prime}=0 \Rightarrow e \text { constante } \\
|\tilde{U}|^{2} & =\Delta^{2}\left(L^{2}-M^{2} / \zeta^{2}\right) /\left|p_{0}-\zeta u_{0}\right|^{2} .
\end{aligned}
$$

a) Filtre asymétrique : $\Delta \neq 0 ; A, D, \zeta, 1 / \zeta$ sont non nuls.

Comme $\zeta$ est imaginaire pur, $L^{2}-M^{2} / \zeta^{2}$ est positif, donc

$$
\tilde{U}=0 \Leftrightarrow p_{0}=\zeta u_{0} \Leftrightarrow Z_{0}=\zeta .
$$

b) Filtre symétrique : $\Delta$ nul, donc $A$ ou $D$ est nul.

$$
\left\{\begin{array} { l } 
{ p = p _ { 0 } } \\
{ u = u _ { 0 } + X D p _ { 0 } }
\end{array} \quad \text { ou } \quad \left\{\begin{array}{l}
p=p_{0}+X A u_{0} \\
u=u_{0} .
\end{array}\right.\right.
$$


L'une au moins des grandeurs $p$ et $u$ a une amplitude constante. Il y a trois cas possibles :

$-A=D=0$ : toute impédance est itérative, et $e^{\prime \prime}=0$.

$-A=0, D \neq 0, \zeta=0: e_{\mathrm{p}}^{\prime \prime}=0$, mais $e_{\mathrm{c}}^{\prime \prime} \neq 0$, et $e_{\mathrm{t}}^{\prime \prime} \neq 0$, sauf dans le cas idéal $\left(Z_{0}=\zeta=0\right)$.

$-A \neq 0, D=0,1 / \zeta=0: e_{\mathrm{c}}^{\prime \prime}=0$, mais $e_{\mathrm{p}}^{\prime \prime} \neq 0$ et $e_{\mathrm{t}}^{\prime \prime} \neq 0$, sauf dans le cas idéal $\left(Y_{0}=1 / \zeta=0\right)$.

4. Le flux d'énergie. - 4.1 I = $\frac{1}{2} \operatorname{Re}\left(p u^{*}\right)=\frac{1}{2}|p|^{2} \operatorname{Re}(Y)=\frac{1}{2}|u|^{2} \operatorname{Re}(Z)$. — La relation linéaire (4) implique : $u(x)=\gamma p(x)+j \frac{\delta}{\varphi} p^{\prime}(x)$ soit $Y(x)=\gamma+j \frac{\delta}{\varphi} \frac{p^{\prime}}{p}$.

Si $I$ est constant, $\gamma$ est imaginaire pur, et $\frac{\delta}{\varphi}$ est réel ; donc :

$$
I=\frac{1}{2}|p|^{2} \operatorname{Re}\left(j \frac{\delta}{\varphi} \frac{p^{\prime}}{p}\right)=-\frac{1}{2} \frac{\delta}{\varphi}|p|^{2} \operatorname{Im}\left(\frac{p^{\prime}}{p}\right)=-\frac{1}{2} \frac{\delta}{\varphi}|p|^{2} \operatorname{Im}\left((\log p)^{\prime}\right)
$$

soit

$$
I=-\frac{1}{2} \frac{\delta}{\varphi}|p|^{2}(\operatorname{Arg} p)^{\prime}
$$

De même, on obtient avec la relation (4bis) : $I=-\frac{1}{2} \frac{\bar{\delta}}{\varphi}|u|^{2}(\operatorname{Arg} u)^{\prime}$.

4.2 BANDE D'ARRÊT IDÉALE $: j \varphi=\alpha .-$

$$
\begin{gathered}
\left\{\begin{array}{l}
p=a \mathrm{e}^{-\alpha x}+b \mathrm{e}^{\alpha x} \\
u=\gamma p+\delta\left(a \mathrm{e}^{-\alpha x}-b \mathrm{e}^{\alpha x}\right)
\end{array}\right. \\
I=\frac{1}{2} \operatorname{Re}\left(\delta\left(a b^{*}-b a^{*}\right)\right)=j \delta \operatorname{Im}\left(a b^{*}\right) .
\end{gathered}
$$

4.3 BANDE PASSANTE IDÉALE : $\varphi$ RÉEL. $-I=\frac{1}{2} \delta\left(|a|^{2}-|b|^{2}\right)$.

4.4 CAS Limite : $\varphi=0$. - Ce qui suit est valable pour $p$, mais s'adapterait sans difficulté pour $u$ :

a) $A \neq 0: \frac{\delta}{\varphi}=-j \frac{\sin \varphi l}{A \varphi}=-\frac{j l}{A} \quad I=\frac{1}{2} \frac{j l}{A}|p|^{2}(\operatorname{Arg} p)^{\prime}$;

b) $A=0 \Rightarrow \Delta=0\left\{\begin{array}{l}p=p_{0} \\ u=u_{0}+j D p_{0} .\end{array}\right.$

Si $D \neq 0: I=\frac{1}{2} \frac{j l}{D}|u|^{2}(\operatorname{Arg} u)^{\prime}$.

\section{Annexe II : ligne sans pertes à caractéristiques variables}

$$
\left.\begin{array}{l}
p=\bar{\gamma} u+\bar{\beta} u^{\prime} \\
u=\gamma p+\beta p^{\prime}
\end{array}\right\}
$$

où $\frac{\gamma}{\beta}=-\frac{\bar{\gamma}}{\bar{\beta}}$ et $\beta, \bar{\beta}, \gamma, \bar{\gamma}$ sont imaginaires purs. Si $\varphi^{2}=\frac{\gamma \bar{\gamma}-1}{\beta \bar{\beta}}$, on a

$$
\left.\begin{array}{rl}
u^{\prime \prime}+u^{\prime} \frac{\bar{\beta}^{\prime}}{\beta}+u\left(\varphi^{2}+\frac{\overline{\gamma^{\prime}}}{\bar{\beta}}\right)=0 \\
p^{\prime \prime}+p^{\prime} \frac{\beta^{\prime}}{\beta}+p\left(\varphi^{2}+\frac{\gamma^{\prime}}{\beta}\right)=0
\end{array}\right\}
$$

1. Equation différentielle satisfaite par $h=L p+M u$. - Le module de $h$ est lié à $e=L^{2}|p|^{2}+M^{2}|u|^{2}$ par la relation $|h|^{2}=e+4 L M I$, où $I=\frac{1}{2} \operatorname{Re}\left(p u^{*}\right)$ est le flux d'énergie, supposé constant. Pour étudier les 
variations de $e$, il suffit donc d'étudier celles de $|h|$. Pour obtenir l'équation satisfaite par $h$, on multiplie les équations du système (9) respectivement par $M$ et $L$, et on doit donc évaluer :

$$
F=L p^{\prime} \frac{\beta^{\prime}}{\beta}+M u^{\prime} \frac{\bar{\beta}^{\prime}}{\bar{\beta}}+\frac{\gamma^{\prime}}{\beta}(L p-M u)
$$

en fonction de $h$ et $h^{\prime}$. D'après (8), $F$ peut se mettre sous la forme suivante :

$$
F=-L p \bar{g}^{\prime}-M u g^{\prime}, \quad \text { où } \quad g=\frac{L}{M \beta}+\frac{\gamma}{\beta} \quad \text { et } \quad \bar{g}=\frac{M}{L \bar{\beta}}+\frac{\bar{\gamma}}{\bar{\beta}} .
$$

Or

$$
h^{\prime}=h g+L p(\bar{g}-g)=h \bar{g}+M u(g-\bar{g}) .
$$

D'où

$$
F=-h^{\prime} \frac{\bar{g}^{\prime}-g^{\prime}}{\bar{g}-g}+h \frac{g \bar{g}^{\prime}-g^{\prime} \bar{g}}{\bar{g}-g}
$$

Finalement, $h$ est solution de :

$$
h^{\prime \prime}-h^{\prime} \frac{\bar{g}^{\prime}-g^{\prime}}{\bar{g}-g}+h\left(\varphi^{2}+\frac{g \bar{g}^{\prime}-g^{\prime} \bar{g}}{\bar{g}-g}\right)=0 .
$$

Posons $\bar{g}-g=G \mathrm{e}^{j \psi} ;$ alors $\frac{\bar{g}^{\prime}-g^{\prime}}{\bar{g}-g}=\frac{G^{\prime}}{G}+j \psi^{\prime}$. D'autre part :

$$
\frac{g \bar{g}^{\prime}-g^{\prime} \bar{g}}{\bar{g}-g}=-\frac{1}{2}(\bar{g}+g)^{\prime}+\frac{1}{2}(\bar{g}+g) \frac{\bar{g}^{\prime}-g^{\prime}}{\bar{g}-g} .
$$

Or $(\bar{g}+g)$ est imaginaire pur; posons $j s=\frac{1}{2}(\bar{g}+g)=\frac{1}{2}\left(\frac{L}{M \beta}+\frac{M}{L \bar{\beta}}\right)$.

L'équation en $h$ s'écrit alors :

$$
h^{\prime \prime}-h^{\prime}\left(\frac{G^{\prime}}{G}+j \psi\right)+h\left(\varphi^{2}-j s^{\prime}+j s\left(\frac{G^{\prime}}{G}+j \psi^{\prime}\right)\right)=0 .
$$

Etude des variations de $|h|:$ Posons $h=\rho \mathrm{e}^{j \theta} \mathrm{e}^{j \frac{1}{2} \psi^{\prime}}$; et prenons la partie réelle de l'équation :

$$
\rho^{\prime \prime}-\rho^{\prime} \frac{G^{\prime}}{G}+\rho\left(-\theta^{\prime 2}+\varphi^{2}-s \psi^{\prime}+\frac{1}{4} \psi^{\prime 2}\right)=0 .
$$

Donc si $\varphi^{2}<s \psi^{\prime}-\frac{1}{4} \psi^{\prime 2} \forall x,\left(\frac{\rho^{\prime}}{G}\right)^{\prime}>0 \quad \forall x$.

Or $\rho^{2}=e+$ constante $\Rightarrow 2 \rho \rho^{\prime}=e^{\prime}: \operatorname{donc}\left(\frac{\rho^{\prime}}{G}\right)^{\prime}>0 \Leftrightarrow\left(\frac{e^{\prime}}{G \rho}\right)^{\prime}>0$.

$$
\text { Si }\left\{\begin{array}{l}
e_{0}^{\prime}<0 \\
\varphi^{2}<s \psi^{\prime}-\frac{1}{4} \psi^{\prime 2} \quad e^{\prime}<0 \quad \forall x<0 .
\end{array}\right.
$$

Donnons enfin une écriture plus simple pour $\psi$ :

$$
\psi=\operatorname{Arctg} \frac{j}{2} \frac{\left(\frac{L}{M \beta}-\frac{M}{L \bar{\beta}}\right)}{-2 \frac{\gamma}{\beta}}=-\operatorname{Arctg} \frac{j}{2}\left(\frac{L}{M \gamma}+\frac{M}{L \bar{\gamma}}\right) .
$$




\section{Bibliographie}

[1] SkIlling, H. H., Electric transmission lines (Mc Graw Hill) 1951.

[2] Brillouin, L. et Parodi, M., Propagation dans les milieux périodiques (Masson, Paris) 1956.

[3] Bouasse, H., Electroptique Ondes Hertziennes (Delagrave, Paris, s.d.) 1915.

[4] Bruhat, G., Optique (Masson, Paris) 1965.

[5] BREKHOVSKIKH, L. M., Waves in layered media (Academic Press, New York) 1960.

[6] Campbell, G. A., Physical theory of the electric wave filter (Bell System Technical J.), vol. I, no 2, 1922.

[7] Johnson, K. S., Circuits de transmission pour communications téléphoniques (Gauthiers-Villars, Paris) 1928.

[8] CAUER, W., Synthesis of linear communication networks (Mc Graw Hill) 1958.

[9] Collin, R. E., Foundations of microwave engineering (Mc Graw Hill) 1966.

[10] Temes, G. C. et Mitra, S. K., Modern filter theory and design (J. Wiley) 1973.

[11] LindSAY, R. B., Mechanical radiation (Mc Graw Hill, New York) 1960.

[12] Slater, J. C., Microwave transmission (Mc Graw Hill) 1942.

[13] Schelkunoff, S. A., Electromagnetic waves (Van Nostrand) 1943.

[14] MORENO, Th., Microwave transmission design data (Mc Graw Hill) 1948.

[15] RaGAN, G. L., Microwave transmission circuits (Mc Graw Hill) 1948.

[16] MarcuwItz, N., Waveguide handbook (Mc Graw Hill) 1951.

[17] Goudet, G. et Chavance, P., Ondes centimétriques (Chiron, Paris) 1955.

[18] BudDEN, K. G., The wave-guide theory of wave propagation (Logos Press, Londres) 1961.

[19] BRILlouIN, L., Wave propagation and group velocity (Academic Press) 1960.

[20] MorSe, P. M. et INGARD, K. U., Theoretical acoustics (Mc Graw Hill) 1968.

[21] Collin, Field theory of guided waves (Mc Graw Hill) 1975.

[22] Standard dictionnary of electrical and electronics terms (I.E.E.E.) 1977.
[23] De Broglie, L., Problèmes de propagation guidée des ondes électromagnétiques (Gauthiers-Villars, Paris) 1951.

[24] JAYNES, E. T., Ghost modes in imperfect waveguides. Proc. I.R.E. 46 (1958) 416.

[25] HuPERT, J. J. et VigiL, J., Evanescent-mode resonator of pure TE mode. Electron. Letters (London) 2 (1966) 251.

[26] HUPERT, J. J., Evanescent-mode coupling in various fields of physics. Am. J. Phys. 41 (1972) 119.

[27] Tung-Lin Tsai et Thomas, G., Analog between optical waveguide system and quantum-mechanical tunneling. Am. J. Phys. 44 (1976) 636.

[28] Crandall, I. B., Vibrating systems and sound (Van Nostrand, New York) 1954.

[29] Olson, H. F., Acoustical engineering (Van Nostrand, Princeton) 1957.

[30] Rocard, Y., Propagation et absorption du son (Hermann, Paris) 1935

[31] RoCARD, Y., Dynamique générale des vibrations (Masson, Paris) 1971.

[32] Crawford, F. S., Waves (Berkeley physics course) (Mc Graw Hill) 1968.

[33] Jouguet, M., Ondes électromagnétiques (2e vol. Propagation guidée) (Dunod-Bordas, Paris) 1973.

[34] Drawin, H. W., Phénomènes de vibration et de propagation (Eyrolles, Paris) 1976.

[35] Kinsler, L. E. et FreY, A. R., Fundamentals of acoustics (John Wiley) 1962.

[36] Stevenson, A. F., General theory of electromagnetic horns. J. Appl. Phys. 22 (1951) 1447.

[37] Benade, A. H. et Jansson, E. V., On plane and spherical waves in horns with nonuniform flare. Acustica 31 (2) (1974) 80 et 31 (4) (1974) 185.

[38] LORD RAYLEIGH, Theory of sound (Dover, New York) 1945, II, § 271.

[39] Fiorito, R. et Überall, H., Resonance theory of acoustic reflection and transmission through a fluid layer. J. Acoust. Soc. Am. 65 (1) (1979) 9.

[40] Kergomard, J., Ondes quasi-stationnaires dans les pavillons avec pertes visco-thermiques aux parois. Acustica 48 (1981) 31. 